\title{
Semi-Lagrangian methods in air pollution models
}

\author{
A. B. Hansen ${ }^{1}$, J. Brandt ${ }^{1}$, J. H. Christensen ${ }^{1}$, and E. Kaas ${ }^{2}$ \\ ${ }^{1}$ National Environmental Research Institute, Aarhus University, Denmark \\ ${ }^{2}$ Niels Bohr Institute, University of Copenhagen, Denmark
}

Received: 26 November 2010 - Published in Geosci. Model Dev. Discuss.: 15 December 2010

Revised: 16 April 2011 - Accepted: 10 June 2011 - Published: 24 June 2011

\begin{abstract}
Various semi-Lagrangian methods are tested with respect to advection in air pollution modeling. The aim is to find a method fulfilling as many of the desirable properties by Rasch and Williamson (1990) and Machenhauer et al. (2008) as possible. The focus in this study is on accuracy and local mass conservation.

The methods tested are, first, classical semi-Lagrangian cubic interpolation, see e.g. Durran (1999), second, semiLagrangian cubic cascade interpolation, by Nair et al. (2002), third, semi-Lagrangian cubic interpolation with the modified interpolation weights, Locally Mass Conserving SemiLagrangian (LMCSL), by Kaas (2008), and last, semiLagrangian cubic interpolation with a locally mass conserving monotonic filter by Kaas and Nielsen (2010).

Semi-Lagrangian (SL) interpolation is a classical method for atmospheric modeling, cascade interpolation is more efficient computationally, modified interpolation weights assure mass conservation and the locally mass conserving monotonic filter imposes monotonicity.

All schemes are tested with advection alone or with advection and chemistry together under both typical rural and urban conditions using different temporal and spatial resolution. The methods are compared with a current state-of-theart scheme, Accurate Space Derivatives (ASD), see Frohn et al. (2002), presently used at the National Environmental Research Institute (NERI) in Denmark. To enable a consistent comparison only non-divergent flow configurations are tested.

The test cases are based either on the traditional slotted cylinder or the rotating cone, where the schemes' ability to model both steep gradients and slopes are challenged.
\end{abstract}

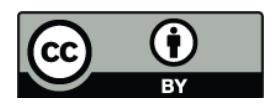

Correspondence to: A. B. Hansen (abu@dmu.dk)
The tests showed that the locally mass conserving monotonic filter improved the results significantly for some of the test cases, however, not for all. It was found that the semiLagrangian schemes, in almost every case, were not able to outperform the current ASD scheme used in DEHM with respect to accuracy.

\section{Introduction}

Semi-Lagrangian (SL) methods (Robert, 1981) do not suffer from the traditional advective CFL-condition severely limiting the maximum possible length of time step, $\Delta t$. These methods have been used widely in the numerical weather prediction (NWP) community, since $\Delta t$ can be defined from accuracy rather than stability considerations. For atmospheric dynamics it turns out that $\Delta t$ can be chosen several times larger than the maximum Courant number without significant loss of accuracy. A main disadvantage of traditional SL methods is that they are not mass conservative when applied to the volume density continuity equation. The SLICE scheme proposed by Zerroukat et al. (2002) and Zerroukat et al. (2004), and the LMCSL scheme by Kaas (2008) are examples of new SL schemes that are mass conserving. There are two aspects of mass conservation: global and local. Global conservation can be imposed by correcting the advected field after determining the global mass loss. However, this does not ensure local conservation of mass. Local mass conservation can be achieved by making the scheme inherently conserving. Both the SLICE and LMCSL are examples of locally mass conserving schemes.

In air pollution modeling, accurate methods are important to be able to model steep gradients in the concentration fields caused by steep gradients in the emission fields and

Published by Copernicus Publications on behalf of the European Geosciences Union. 
by non-linear atmospheric chemistry. In chemical transport models it is crucial that no negative values are generated. Not only are they "unphysical" but they also cause the chemical part of the model to break down. The present advection scheme in the Danish Eulerian Hemispheric Model (DEHM) applied to the mixing ratio version of the continuity equation is the ASD. ASD is very accurate compared to other schemes, see Dabdub and Seinfeld (1994). The ASD scheme can, however, create problems near sharp gradients. Spurious waves known as the Gibbs phenomenon can occur when such features are present, and they can result in unphysical negative values.

In this work a new numerical method is proposed for solving the volume density continuity equation. The aim of this method is to fulfill as many of the desirable properties by Rasch and Williamson (1990) and Machenhauer et al. (2008) as possible. The new method is based on the semi-Lagrangian cubic interpolation combined with cascade interpolation developed by Nair et al. (2002), the modified interpolation weights suggested by Kaas (2008) and the locally mass conserving monotonic filter by Kaas and Nielsen (2010). An SL forecast of the prognostic variable $\psi$ at time step number $n+1$ and Eulerian grid point $i$, i.e. $\psi_{i}^{n+1}$, is obtained via Lagrangian or spline interpolation from Eulerian points at time step $n$ surrounding the departure point of that trajectory ending up in grid point $i$ after one time step. The interpolation weights depend on the distance to the departure point. In the NWP community it has been found that third order (cubic or cubic spline) interpolation provide a good compromise between accuracy and numerical cost. For application in an $N$-dimensional problem it is possible to apply so-called cascade interpolation, i.e. the $N$-dimensional interpolation problem is converted into $N$ one-dimensional interpolation problems, which simplifies the calculations. In the cascade method proposed here $N=2$. In a non-divergent problem the sum of the $N$-dimensional interpolation weights given off from a particular Eulerian point $j$ to all surrounding departure points should equal one, see Sect. 4.3.4. Generally, however, in the semi-Lagrangian approach, they do not. Therefore the raw interpolation weights are normalised so that the sum of the weights given off from any Eulerian point, $j$, at time level $n$ equals the area/volume represented by grid cell $j$. Thereby all mass, of each Eulerian point of the domain is "used" once and only once. This ensures that mass is neither created nor lost during interpolation. Which ensures local and global mass conservation. It still remains to be seen whether these modified interpolation weights also will perform better when used in real applications.

The overall purpose of this work is to develop, implement, and test selected numerical advection algorithms based on the semi-Lagrangian approach. This has been done by developing a Fortran code for all methods in order to ensure the exact same conditions for comparison. This leads to the following scientific hypothesis which will be tested in the present study.

\section{Hypothesis}

The classical cubic semi-Lagrangian approach, including different combinations of cascade interpolation, the mass conserving modified interpolation weights and a locally mass conserving monotonic filter, performs better than the present advection scheme used in DEHM based on Accurate Space Derivatives when tested on a non-divergent modified rotation test including chemistry.

In order to test this hypothesis an advection algorithm has been developed with the possibility to include and exclude the different features of the different schemes. In this way the effect of the different methods can be tested separately or in combination. The algorithm is tested on "solid body rotation" of a slotted cylinder as described by Zerroukat et al. (2002) and Sun and Yeh (1997) and of a rotating cone by Molenkamp (1968) and Crowley (1968).

The present article is composed as follows. In the following section, Sect. 3, the background for this project is described along with the processes considered in air pollution modelling. In Sect. 4 the theory used, for building the different advection schemes tested in this work, is described. The various advection schemes tested in the present study are described in detail in Sect. 5. In Sect. 6, the obtained results are shown. Lastly, a discussion of the results, the conclusion and future aspects are given in Sect. 7.

\section{Background}

In air pollution models the equations describe the atmospheric transport and chemistry as well as diffusion and dry and wet deposition. If the purpose is to model the chemical development and transport of e.g. $\mathrm{O}_{3}$ and $\mathrm{NO}_{2}$, one would not obtain good results unless other compounds which interact with these species are included. However, a model usually contains a limited number of species (typically 30 to 80 ) rather than every chemical constituent that exists in the atmosphere. Many of the left out species would not be relevant, in this case for $\mathrm{O}_{3}$ and $\mathrm{NO}_{2}$, but would just complicate the calculations.

In order to decide which processes are relevant, one must consider the time scale of the integration. In addition to chemical reactions and transport, including diffusion, in the atmosphere, it is also important to consider sources and sinks, e.g., emissions into the atmosphere and dry and wet deposition of species in the atmosphere to the surface.

\subsection{Air pollution models}

As stated above, different equations are needed to solve different problems. In the case of air pollution models, the mixing ratio continuity equation can be used to model the relevant processes in the atmosphere. To solve this equation numerically it can be advantageous to split it into submodels. These submodels are solved individually and they describe, 
in DEHM, first, 3-D advection, second, diffusion in three directions and last chemistry, wet deposition and emissions. The submodels can be solved using the same or different numerical algorithms. Furthermore, the advection might be split into a two-dimensional horizontal advection and a onedimensional vertical advection, since the horizontal and vertical advection are different in their characteristics, e.g. the wind components $u, v$ and $w$. For a more detailed discussion the reader is referred to Frohn et al. (2002).

\subsection{Mixing ratio versus volume density}

The chemistry component of air pollution models requires as input (and output) the various mixing ratios $c_{i}$ of the individual chemical species, $i=1,2, \ldots, q$, where $q$ is the number of chemical constituents included in the model. Therefore the mixing ratio continuity equation, which is identical to the advection equation with source and sink terms, is used directly as prognostic equation for transporting the individual species in most air pollution models such as DEHM. Only considering the processes of atmospheric transport and diffusion the Eulerian formulation of this equation reads:

$\frac{\partial c_{i}}{\partial t}=-\boldsymbol{U} \cdot \nabla c_{i}+\operatorname{diffusion} / \mathrm{mixing}$

where $\boldsymbol{U}$ represents the velocity vector. In the present study we are, however, comparing the performance of numerical algorithms directly solving Eq. (1) (i.e. DEHM) with other methods where the full continuity equation, i.e. the volume density continuity equation, is solved for both dry air and for each individual chemical species. For such methods the mixing ratio at any instant must be obtained as $c_{i}=\rho_{i} / \rho$, where $\rho_{i}$ and $\rho$ are the volume densities of the individual species and of the dry air, respectively. The Eulerian volume density continuity equation for chemical species no. $i$ reads:

$\frac{\partial \rho_{i}}{\partial t}=-\boldsymbol{U} \cdot \nabla \rho_{i}-\rho_{i} \nabla \cdot \boldsymbol{U}+$ diffusion $/$ mixing.

The Lagrangian version of this equation reads:

$$
\frac{d \rho_{i}}{d t}=-\rho_{i} \nabla \cdot \boldsymbol{U}+\text { diffusion/mixing }
$$

In air pollution models employing Eq. (2) or Eq. (3) it is necessary at each time to convert $\rho_{i}$ to $c_{i}$, i.e. $c_{i}=\rho_{i} / \rho$, before calling the modelling components dealing with chemistry, and to convert back again to obtain the updated values of $\rho_{i}$. In the abcense of divergence, it is noted that the prognostic equations for $c_{i}$ and $\rho_{i}$ are identical.

\subsection{Numerical treatment of the advection in DEHM}

There exist many different numerical methods to solve the advection equation, each with their own advantages and disadvantages. The challenge is to find the most suitable scheme for the problem considered, see e.g. Brandt (1998). For example, in Brandt et al. (1996a), four different Eulerian methods have been tested, namely, the up-wind method, finite elements, the Bott scheme and Holm's algorithm. The algorithms were tested for their ability to model advection when combined with chemistry.

The upwind method is computationally very cheap, but it produces a lot of artificial numerical diffusion, which makes it unsuitable for use in air pollution models.

The finite element scheme is relatively fast and it can work on irregular grids. However, this scheme has a higher sensitivity than others when considering sharp gradients, see Brandt (1998).

The Bott scheme, by Bott (1989), is a flux based method. It was developed to make a conservative and positive definite advection scheme with a limited amount of numerical diffusion. The Bott scheme is used in many regional models within Europe, e.g. the Danish Meteorological Institute uses this scheme in Enviro-HIRLAM.

The method is considered computationally very efficient, see Bott (1989), because it is explicit and forward-in-time. In most applications it is, however, only first order accurate in time and space. Small time steps are used in order to prevent violation of the CFL criterion. To reduce the numerical diffusion, up to tenth order polynomials are used. High order polynomials might introduce negative values, however, this is limited by normalising the fluxes and suppression of negative values. This implies considerable numerical diffusion near sharp gradients.

The Bott and Holm schemes produce smooth concentration fields with no negative concentrations, but they are in general more expensive than the upwind or finite element schemes. The Holms scheme is the best one among these methods. However, it is also the most the most timeconsuming Brandt et al. (1996a).

In DEHM, the ASD scheme combined with a Forester filter was chosen, because it was proven to be the best performing scheme amongst the Smolarkiewicz method, the Galerkin finite element method, the numerical method of lines, the accurate space derivative method, the Bott method and the Emde method, see Dabdub and Seinfeld (1994).

\subsection{General description of DEHM}

The Danish Eulerian Hemispheric Model (DEHM) is an Eulerian atmospheric chemistry transport (ACT) model in three dimensions. The model was originally developed for the study of long-range transport of $\mathrm{SO}_{2}$ and $\mathrm{SO}_{4}^{2-}$ into the Arctic, see Christensen $(1995,1997)$. The present version of the model contains 58 photo-chemical species, 9 primary emitted particles, 14 persistent organic pollutants, and 7 mercury species.

DEHM is an "off-line" atmospheric chemical transport model. This means that simulations of the driving winds, precipitation etc. are not part of DEHM. These fields are 
imported from other data sources and interpolated in time and space to the grid used in DEHM. The meteorological data used as input to DEHM is produced by the MM5v3 model run at NERI, the model uses the same grid definition as DEHM and writes output every hour. This means that there is no spatial interpolation of the data and errors from temporal interpolation of the data are minimized.

\subsubsection{The mixing ratio equation of continuity}

The mixing ratio continuity equation describes the change in mixing ratio of a certain chemical species over time. Expressed using the Eulerian approach, it contains terms describing the spatial gradients in concentration as well as diffusion, both horizontally and vertically. Furthermore, terms accounting for sources and sinks including chemistry, wet deposition and emissions are included. In DEHM, the following equation is used:

$$
\begin{aligned}
\frac{\partial c_{i}}{\partial t}= & -\left(u \frac{\partial c_{i}}{\partial x}+v \frac{\partial c_{i}}{\partial y}+\dot{\sigma} \frac{\partial c_{i}}{\partial \sigma}\right) \\
& +K_{\mathrm{x}} \frac{\partial^{2} c_{i}}{\partial x^{2}}+K_{\mathrm{y}} \frac{\partial^{2} c_{i}}{\partial y^{2}}+\frac{\partial}{\partial \sigma}\left(K_{\sigma} \frac{\partial c_{i}}{\partial \sigma}\right) \\
& +E_{i}(x, y, \sigma, t)-\Lambda_{i} c_{i} \\
& +Q_{i}\left(c_{1}, c_{2}, \ldots, c_{q}\right) \quad(i=1,2, \ldots, q)
\end{aligned}
$$

Here, $c_{i}$ is the mixing ratio of chemical species $i$. The $\sigma$ coordinate is a terrain following coordinate, where the pressure is normalized by the surface pressure. $\dot{\sigma}$ is the vertical wind speed in this coordinate system. $u, v$, and $\dot{\sigma}$ represent the $\mathrm{x}-, \mathrm{y}-$, and $\sigma$-component of the wind, respectively. Likewise $K_{\mathrm{x}}, K_{\mathrm{y}}$, and $K_{\sigma}$ are the $\mathrm{x}-, \mathrm{y}-$, and $\sigma$-components of the diffusion coefficients. The horizontal diffusion coefficients, $K_{\mathrm{x}}$ and $K_{\mathrm{y}}$, are assumed to be constant, the vertical diffusion coefficient is both temporal and spatial dependent. $E_{i}$ is the emission of a given species, $\Lambda_{i}$ is the wet deposition scavenging coefficient and $Q_{i}$ represents the chemistry. Dry deposition is applied as the lower boundary condition for the vertical dispersion. $i$ counts the $q$ different chemical species.

Using first order splitting various methods can be used to solve the different components of the equation.

\subsubsection{Chemistry}

In this work the extensive chemical version of DEHM, which includes 58 species is used. Some of the species included are $\mathrm{SO}_{\mathrm{x}}, \mathrm{NO}_{\mathrm{x}}, \mathrm{NH}_{\mathrm{x}}, \mathrm{O}_{3}, \mathrm{VOCs}$ and secondary inorganic particulates (Frohn et al., 2003). The chemical scheme was based on a scheme with 51 species presented in Flatøy and Hov (1996), which was an ozone chemistry scheme with most of the important inorganic species as well as the most abundant hydrocarbons (explicit treatment of alkanes with up to four carbon atoms, longer alkanes lumped, explicit treatment alkenes with up to three atoms, again, longer alkenes lumped, xylene, toluene and isoprene). There has been added reactions to extend the chemistry to be used for eutrophication issues by using ammonium chemistry based on the old EMEP acidification model and adding reactions in order to extend to acidification issues by using aqueous chemistry based on Jonson et al. (2000). The scheme contains 120 chemical reactions where 17 are photolysis reactions calculated by the Phodis routine (Kylling et al., 1998) depending on sun-angle, altitude, Dobson unit and 3-D cloud cover. The used chemical scheme is quite similar to the EMEP scheme described in Simpson et al. (2003).

In this work the sun angle is prescribed to correspond to the diurnal variation of a summer day. The chemistry can simulate either urban or rural conditions, by changing the initial concentrations of $\mathrm{NO}$ and $\mathrm{NO}_{2}$.

The chemistry is very stiff and uses a time step much smaller than the time step of the advection. The chemistry is calculated using the Euler Backward Iterative method to calculate the first time step of the chemistry. Afterwards, the two-step method is used for the rest of the time steps, see Frohn (2004). This procedure is used because the two-step method needs two initial time steps to be able to perform the time integrations.

\section{Theory}

In this work the semi-Lagrangian cubic interpolation (Durran, 1999), cascade interpolation (Nair et al., 2002), modified interpolation weights (Kaas, 2008) and the locally mass conserving monotonic filter (Kaas and Nielsen, 2010) are tested individually and combined. The focus is on fulfilling as many of the desirable properties (Rasch and Williamson, 1990; Machenhauer et al., 2008) as possible, and test the method on a slotted cylinder (Zerroukat et al., 2002) and the rotating cone (Molenkamp, 1968; Crowley, 1968).

In this section the theory behind the various equations and methods used is described. First, in Sect. 4.1 the form of the continuity equation used here is described. In Sect. 4.2 the desirable properties for advection schemes are described.

The advection algorithm used in DEHM is described in Sect. 4.4, including ASD and the Bartnicki Filter in Sect. 4.4.1 and the Forester filter in Sect. 4.4.2. A description of the test cases is given in Sect. 4.5.

\subsection{The continuity equations}

In the present work, only horizontal advection and chemistry is considered. Then the equations for mixing ratio take the form:

$$
\begin{array}{r}
\frac{\partial c_{i}}{\partial t}=-\left(u \frac{\partial c_{i}}{\partial x}+v \frac{\partial c_{i}}{\partial y}\right)+Q_{i}\left(c_{1}, c_{2}, \ldots, c_{q}\right) \\
(i=1,2, \ldots, q) .
\end{array}
$$

Here, $c_{i}$ is the concentration of the chemical species $i . u$ and $v$ represent the $\mathrm{x}$ - and $\mathrm{y}$-component of the wind, respectively. 
Note that we have omitted source, deposition and horizontal diffusion terms since, in the present study, we are only considering an idealised case and we only look at solid body rotation where the true horizontal diffusion - by definition is zero.

Equation (6) is split into a submodel for transport (the first tendency term on the right hand side) and a submodel dealing with chemical reactions (the last tendency term, $Q_{i}$, on the right hand side).

As explained in Sect. 3.2 the dynamical prognostic equations to be solved in the submodel for transport, in case of the new numerical schemes, are the volume density continuity equations:

$$
\begin{array}{r}
\frac{d \rho_{i}}{d t}=-\rho_{i}\left(\frac{\partial u}{\partial x}+\frac{\partial v}{\partial y}\right) \\
(i=1,2, \ldots, q) .
\end{array}
$$

We also need to solve the continuity equation for the density of dry air

$$
\frac{d \rho}{d t}=-\rho\left(\frac{\partial u}{\partial x}+\frac{\partial v}{\partial y}\right)
$$

For the new schemes we can then calculate $c_{i}=\rho_{i} / \rho$. Once $c_{i}$ is estimated from Eqs. (7) and (8) we can apply the chemical submodel just as in the case of the DEHM model, i.e. the effect of tendencies due to the last term in Eq. (6). The test cases presented in Sect. 4.5 are non-divergent. In this special case the atmospheric transport Eqs. (7) and (6) excluding the chemical part, are identical. For the new schemes we have therefore not performed the division by $\rho$, i.e. we have simply assumed $\rho=1$ and, thus, formally that $\rho_{i}=c_{i}$.

\subsection{Desirable properties}

When modelling the continuity equation, there are certain desirable properties the results should fulfill. Rasch and Williamson (1990) defined seven desirable properties: accuracy, stability, computational efficiency, transportivity and locality, shape-preservation, conservation, and preservation of linear correlation between constituents. According to Machenhauer et al. (2008) three additional properties are desirable. These are consistency of the discretization, compatibility, and preservation of constancy. The ultimate goal of numerical methods is to fulfill all of these properties simultaneously, but so far this has not been possible. Therefore one should try to satisfy as many of these properties as possible. Below it will be described what is meant by each of these desirable properties. The formulation of Machenhauer et al. (2008) is used.

In any numerical programming, high accuracy is the primary goal, and often includes most of the properties listed above. However, when modelling flow with steep gradients or shocks, the order of accuracy found from Taylor series expansions, see Durran (1999), might have nothing to do with the accuracy of the particular problem. Instead the standard error measures $l_{1}, l_{2}$, and $l_{\infty}$ are widely used by the meteorological community for idealised test cases, see e.g., Machenhauer et al.:

$$
\begin{aligned}
l_{1} & =I(|\phi-\psi|) / I(|\psi|), \\
l_{2} & =\sqrt{I(\phi-\psi)^{2}} / \sqrt{I(\psi)^{2}}, \\
l_{\infty} & =\max [|\phi-\psi|] / \max [|\psi|] .
\end{aligned}
$$

Here $\phi$ is the numerical solution, $\psi$ is the exact solution if one such exists, otherwise a reference of high-resolution is used. $I(\cdot)$ denotes the integral over the entire domain. The first two error measures are measures of the global "distance" between the numerical and the true solution. The $l_{\infty}$ error measure gives the normalised maximum deviation between $\phi$ and $\psi$ over the entire domain. In relation to undershooting and overshooting, normalised minimum and maximum values of the numerical solutions are also used to indicate errors.

Stability ensures that the numerical solution does not blow up with time. It can be achieved by adding filters to the numerical method; in Lagrangian models, this is usually not a severe problem. In global Eulerian models, the stability problem is most severe near the poles. As a measure for stability in Eulerian models, the CFL condition and the Courant number are used.

For the one-dimensional case, the Courant-FriedrichLewy (CFL) condition is given by:

$$
\frac{u \cdot \Delta t}{\Delta x}<\gamma
$$

Where $u$ is the velocity, $\Delta t$ the time step, $\Delta x$ the grid resolution, and $\gamma$ is a constant depending on the specific advection algorithm. The number

$C=\frac{u \cdot \Delta t}{\Delta x}$

is called the Courant number.

Computational efficiency refers to the fact that the program should be fast when run. When considering parallel computing it is important to note whether the parameterisation is local, as SL, or global, like ASD. Too much communication between the nodes tend to slow down the calculations.

Locality refers to the domain of dependence. The true solution's domain of dependence should lie within the domain of dependence of the numerical solution. This is also referred to as the CFL condition, see Fig. 2.1 from Durran (1999). Transportivity is of special interest in transport models. Rasch and Williamson (1990) writes "an algorithm possesses the transportive property if a perturbation in the field is advected only down wind".

In pure advection, no alteration should be made to a scalar field, that is, no new extrema must be generated during the numerical approximation, only the physical extrema should be reproduced. This is referred to as shape preservation. 
Such new unphysical extrema might generate negative mixing ratios or too high values. A positive definite scheme prevents negative values in the solution. Schemes preventing generation of new extrema are referred to as monotonic.

When modelling the continuity equation, it is very important that mass is conserved. Changes in the global mass budget by non-conserving methods over long time integrations reduce the accuracy of the model. To avoid such problems finite-volume methods can be used, see e.g. Machenhauer et al. (2008). Numerically ASD is a mass conserving method, using the surface pressure from a meteorological model to correct the horizontal wind components which ensures mass conservation.

The consistency of the discretization property concerns the coupling between the volume density continuity equation for air as a whole and that for the individual tracer constituents. If different numerical techniques, different time stepping and/or different spatial resolution is used for the air as a whole and for the tracer components, one may introduce mixing ratios $\left(c_{i}=\rho_{i} / \rho\right)$ which are inconsistent. By this is meant that if one of the tracers initially and deliberately is chosen to be identical to the volume density of the whole air then the mixing ration for this special tracer will not continue to have mixing ratio equal to 1 .

The compatibility property states that in models based on the volume density continuity equation the mixing ratio, which is recovered as $c_{i}=\rho_{i} / \rho$, should be limited by the mixing ratios in grid cells which are neighbours to the upstream departure point. In Eulerian finite volume models the Courant number is always less than one and therefore the limits are defined from the grid cells neighboring the cell to be forecasted.

In non-divergent flow, it is desirable for the scheme to be able to preserve a constant tracer field. This is trivial for traditional semi-Lagrangian methods based on the mixing ratio version of the continuity equation since the prognostic equation does not explicitly contain the divergence of the velocity field. For models based on the volume density version of the continuity equation this is, however, not always the case.

In chemical atmospheric models it is crucial that tracer correlations are conserved, since these have great influence on speed and balances of chemical reactions. In this work the focus is on accuracy and mass conservation.

\subsection{Methods}

This section describes the methods considered in the present work. In Sect. 4.3.1 the traditional semi-Lagrangian method is described along with the properties of the method. Section 4.3.3 describes cascade interpolation, followed by the modified interpolation weights in Sect. 4.3.4. The locally mass conserving monotonic filter and calculation of trajectories are described in Sect. 4.3.5 and Sect. 4.3.6, respectively.

\subsubsection{The semi-Lagrangian method}

To count steps in the spatial dimensions and time, various letters in subscript and superscript are used. In the $\mathrm{x}$-direction $i, k$, and $p$ are used. In the y-direction $j, l$, and $q$ are used and to count time $n$ and $n+1$ are used.

When indexing the weights, e.g. $\alpha_{i, j}$, the first subscript refers to the point of which $\alpha$ is the value, and the second subscript refers to the point which is being calculated.

In the Lagrangian approach, the equations describe the flow following the motion of a particle or air parcel. At the initial time step, a uniform grid is chosen and the particles are followed while integrating forward in time. This might be cumbersome, because the particles in practical problems seldom stay evenly distributed. To avoid this, the semiLagrangian method was introduced. In this method, a completely new grid is chosen at every time step. This new grid consists of the particles arriving at the end of the time step. The departure point of each particle is then found integrating the equations backward in time one step.

Here, to start with, the scalar advection equation is derived in one dimension for constant velocity using linear interpolation, and hereafter it is extended to cubic interpolation. Following the notation of Durran, considering a passive tracer, the advection equation can be written as (Equation 6.4, Durran, 1999):

$\frac{\phi\left(x_{i}, t^{n+1}\right)-\phi\left(\tilde{x}_{i}^{n}, t^{n}\right)}{\Delta t}=0$,

here, $\tilde{x}_{i}^{n}$ refers to the departure point of the particle and $\left(x_{i}, t^{n+1}\right)$ is the arrival point of the particle. Using $\boldsymbol{U}$ to denote the constant velocity the backward calculation of the trajectory may be written as:

$\tilde{x}_{i}^{n}=x_{i}-\boldsymbol{U} \Delta t$.

If $\boldsymbol{U}$ is positive and $p$ is defined as the integer part of $\boldsymbol{U} \Delta t / \Delta x, \tilde{x}_{i}^{n}$ will be in the interval between $x_{i-p-1}$ and $x_{i-p}$, see Fig. 6.1 of Durran (1999).

The non-integer part of $\boldsymbol{U} \Delta t / \Delta x$ is called $\alpha$, and defined as:

$\alpha \equiv \frac{x_{i-p}-\tilde{x}_{i}^{n}}{\Delta x}$.

Writing $\phi\left(x_{i}, t^{n}\right)$ as $\phi_{i}^{n}$, the scalar advection equation for constant velocity, using linear interpolation to approximate the departure point, is (Eq. 6.5 of Durran):

$\phi_{i}^{n+1}=(1-\alpha) \phi_{i-p}^{n}+\alpha \phi_{i-p-1}^{n}$.

This is stable for arbitrarily large time steps, because the true domain of dependence is included in the numerical domain of dependence caused by the backward calculation of trajectories, which ensures that the CFL condition is not violated.

In spite of the fact that semi-Lagrangian schemes are stable for arbitrarily large time steps, large time stepping is not 
always advantageous. In case of air pollution modeling or when considering, e.g., accidental releases, time steps over several grid points can introduce errors in emissions and deposition. The species which would have been emitted into the atmosphere continuously will instead appear discontinuous with mass of the emitted species only in some grid points. The same happens when considering deposition.

\subsubsection{Cubic interpolation}

The linear interpolation of the scalar advection equation is too diffusive to be useful (Durran, 1999, p. 308), so to approximate equations with smooth solutions higher-order semi-Lagrangian interpolation are used, with the same conditions for $\boldsymbol{U}, q$, and $\alpha$ as above, a cubic interpolation can be made by using the four closest grid-points, (Eq. 6.12 of Durran, 1999):

$$
\begin{aligned}
\phi\left(\tilde{x}_{i}^{n}, t^{n}\right) & =-\frac{\alpha\left(1-\alpha^{2}\right)}{6} \phi_{i-p-2}^{n} \\
& +\frac{\alpha(1+\alpha)(2-\alpha)}{2} \phi_{i-p-1}^{n} \\
& +\frac{\left(1-\alpha^{2}\right)(2-\alpha)}{2} \phi_{i-p}^{n} \\
& -\frac{\alpha(1-\alpha)(2-\alpha)}{6} \phi_{i-p+1}^{n}
\end{aligned}
$$

In two spatial dimensions, this is called bi-cubic interpolation, $\beta$ is the non-integer part of the displacement in the y-direction. $\alpha$ and $\beta$ are now defined as $(\boldsymbol{U} \Delta t-p) / \Delta x$ and $(\boldsymbol{V} \Delta t-q) / \Delta y$, respectively, where $\boldsymbol{U}$ is the velocity in the x-direction, $\boldsymbol{V}$ is the velocity in the y-direction. $p=\operatorname{int}(\boldsymbol{U} \Delta t / \Delta x)$ and $q=\operatorname{int}(\boldsymbol{V} \Delta t / \Delta y)$, where int() denotes the integer part.

Using von Neumann stability analysis, this scheme can be shown to be unconditionally stable, see e.g. Kaas (1987).

\subsubsection{Cascade interpolation}

In cascade interpolation, the two-dimensional advection is split into two one-dimensional interpolations. This method is more efficient than traditional semi-Lagrangian interpolation. In cubic cascade interpolation $2 \times 4$ departure points are used instead of the $4 \times 4$, or 16 departure points, used in traditional semi-Lagrangian bi-cubic interpolation.

After the Lagrangian true departure points are found, the interpolation is split into three steps. First, using linear interpolation in the $\mathrm{y}$-direction the displacements in the $\mathrm{x}$ direction are determined. Second, the new x-displacements are used to perform the advection in the $\mathrm{x}$-direction; this result is termed $\phi$ intermediate. Last, $\phi$ intermediate is used to calculate the advection in the y-direction. Both in the second and third step one-dimensional cubic interpolation is used, this corresponds to the $2 \times 4$ departure points. However, the two sets of departure points are not the same. The first four points are the values from the previous time step, but the last four are the values from the intermediate time step, when the interpolation has been performed only in one direction. Nair et al. (2002) have proposed a scheme combining conservative finite-volume methods with semi-Lagrangian cascade interpolation. Cascade interpolation can be used to reduce a twodimensional problem to two one-dimensional remappings.

Cascade interpolation can be explained by considering a rigid uniform grid (Eulerian or Cartesian), see Fig. 1 of Nair et al. (2002). In the $\lambda$ and $\mu$ directions, the distance between two neighbouring grid points is $\Delta \lambda$ and $\Delta \mu$, respectively. This is the arrival grid. For each intersection of an Eulerian longitude and an Eulerian latitude, each Eulerian grid point $\left(\lambda_{i}, \mu_{j}\right)$, there is a corresponding upstream Lagrangian point $\left(\lambda_{i j}, \mu_{i j}\right)$, the departure point. As shown in the figure, four neighbouring grid points, whether Eulerian or Lagrangian, bounding a rectangular region define the respective cells of the grid system.

The Lagrangian system of grid cells corresponds to the arrival grid at the previous time step. The intersection of a Lagrangian latitude and an Eulerian longitude, is defined as an intermediate grid point.

In the cascade interpolation the intermediate grid is generated first. This can be calculated using one-dimensional cubic semi-Lagrangian interpolation. For the calculation the four nearest points along the Lagrangian latitude, $\left\{\lambda_{i-1, j}, \lambda_{i, j}, \lambda_{i+1, j}, \lambda_{i+2, j}\right\}$, are used. The entire computational domain is spanned exactly by the intermediate cells, however, for efficiency in the computation, the horizontal lines within each Eulerian grid cell approximate the Lagrangian latitudes. These approximated Lagrangian latitudes are defined as $\tilde{\mu}_{\hat{j}}=\left(\tilde{\mu}_{i, j}+\tilde{\mu}_{i+1, j}\right) / 2$ and the distance between two adjacent grid points $\tilde{\mu}_{\hat{j}+1}$ and $\tilde{\mu}_{\hat{j}}$ is given by $\Delta \tilde{\mu}_{\hat{j}}=\tilde{\mu}_{\hat{j}+1}-\tilde{\mu}_{\hat{j}}$. The approximated Lagrangian longitude is calculated in a similar way. The cells made up by the approximated Lagrangian latitudes and longitudes are called the computational cells. Please, note again that the cells cover the entire domain, but do not overlap.

The remapping is now performed, one dimension at a time. First mass is transferred in the $\mu$ direction from Eulerian to intermediate cells. This is done by calculating the average density $\bar{h}_{j}$. Looking at one column at a time, the dependence on $i$ is omitted. The initial mass in the Eulerian grid is given by $M_{i j}=\bar{\rho}_{i j}^{n} \Delta \mu_{j} \Delta \lambda_{i}$ with $\bar{\rho}_{i j}^{n}$ as the cell-averaged density at the $n$-th time step, and the area of the cell $\Delta \mu_{j} \Delta \lambda_{i} . \bar{h}_{j}$ is given by the initial mass divided by the cell width of the $j$-th cell, $\bar{h}_{j}=M_{i j} / \Delta \mu_{j}$. This average density is used to construct piecewise parabolic profiles of the vertical columns. The mass calculated above is used to find the average density per $\Delta \lambda_{i}$ in the intermediate cells. This is used to fit piecewise parabolic profiles, so that the mass is represented by the area under the curve. The average density of the arrival cells at the next time step $(n+1)$ is determined using the newly calculated mass. For a more detailed description, see Nair et al. (2002). 


\subsubsection{Modified interpolation weights}

When used for solving the volume density version of the continuity equation traditional upstream interpolated semiLagrangian schemes do not conserve mass, but Kaas (2008) suggested a new method inspired by CISL (Cell Integrated Semi-Lagrangian) schemes, mentioned above, introducing modified upstream SL-weights. This scheme is locally mass conserving and therefore called the LMCSL (Locally Mass Conserving Semi-Lagrangian) scheme. In addition to calculating the traditional semi-Lagrangian interpolation weights, these are also used to calculate modified upstream SL-weights. The idea is based on the concept of partition of unity, since the traditional interpolation weights, which should sum to one in the case of no divergence and homogenous resolution, are weighted to ensure mass conservation.

Using the notation of Kaas (2008), the continuity equation for volume density can be written as:

$\rho_{k}^{n+1}=\sum_{l=1}^{K} \hat{w}_{k, l} \rho_{l}^{n}$,

with

$\hat{w}_{k, l}=\frac{A_{l}}{A_{k}} \frac{w_{k, l}}{\sum_{m=1}^{K} w_{m, l}}$.

Here $w_{k, l}$ corresponds to the semi-Lagrangian weight and $A_{k}$ represents the area, in two dimensions, or volume, in three dimensions, of the $k$-th Eulerian grid point. The modified interpolation weights include the divergence. This formulation can be used for any order of interpolation. It can be shown that the total mass at time step $n+1$ equals that at time step $n$ for a periodic domain:

$$
\begin{aligned}
\sum_{k=1}^{K} A_{k} \rho_{k}^{n+1} & =\sum_{k=1}^{K} \sum_{l=1}^{K} A_{k} \hat{w}_{k, l} \rho_{l}^{n} \\
& =\sum_{k=1}^{K} \sum_{l=1}^{K} \frac{A_{l} w_{k, l}}{\sum_{m=1}^{K} w_{m, l}} \rho_{l}^{n} \\
& =\sum_{l=1}^{K} A_{l} \frac{\sum_{k=1}^{K} w_{k, l}}{\sum_{m=1}^{K} w_{m, l}} \rho_{l}^{n} \\
& =\sum_{l=1}^{K} A_{l} \rho_{l}^{n},
\end{aligned}
$$

at the first equality sign Eq. (19) has been used to go one time step back, at the next, Eq. (20) has been used to substitute $\hat{w}_{k, l}$, at the third the terms are rearranged and at the last equality sign it is used that $\sum_{k=1}^{K} w_{k, l}=1$. Hence it is shown that the scheme has formal mass conservation.

\subsubsection{Locally mass conserving monotonic filter}

The filter used here is the locally mass conserving and antidiffusive monotonic filter for use in semi-Lagrangian models by Kaas and Nielsen (2010). Plainly speaking, the filter redistributes mass in all points bringing the forecast as close as possible to a monotonic anti-diffused but non-massconserving forecast. First the "real" forecast of high accuracy, $\rho$, in this case cubic interpolation, and a low resolution linear forecast, $\rho_{\mathrm{L}}$, are calculated. In addition to the two forecasts the filter also requires a condition to achieve monotonicity: the new value in any given point must not exceed the maximum or minimum values, $\rho_{\min }$ and $\rho_{\max }$, of the grid points surrounding the departure points. Here for two dimensions, four points are considered when evaluating $\rho_{\text {min }}$ and $\rho_{\text {max }}$.

The filter works by first applying an anti-diffusive filter on the non-filtered forecast and constraining these values to be monotone and mass conserving. The result is termed a target value forecast.

The mathematical description of the filter is given by the following Eqs. (22)-(31). $\rho_{\min }$ and $\rho_{\max }$ are given by Eq. (22) under the assumption of a non-divergent flow. The minimum and maximum values are found from the surrounding grid points of the departure points, denoted by $l$.

$$
\begin{gathered}
\rho_{\min k}^{n+1}=\min _{l}\left(\rho^{n}\right) \\
\rho_{\max k}^{n+1}=\max _{l}\left(\rho^{n}\right),
\end{gathered}
$$

$k$ as above represents a general grid point index, i.e. $k=$ $1, \ldots, K$, where $K$ is the total number of grid points. The linear anti-diffusioned forecast is calculated using the following equation.

$\rho_{A}=\rho_{k}+\alpha_{k}\left(\rho_{k}-\rho_{L_{k}}\right)$

$\alpha_{k}$ is the strength of the anti-diffusion - or diffusion in the case where $\alpha_{k}<0$. In this study $\alpha_{k}$ is determined from:

$\alpha_{k}=\max \left[0.09,-0.246+6.64 \sigma_{k}-12.29 \sigma_{k}^{2}\right]$

In Eq. (24) $\sigma$ is a parameter determining the local scale of $\rho$ with small scales corresponding to high values of $\sigma$. The coefficients in Eq. (25) have been obtained empirically, see details in Kaas and Nielsen (2010).

$\sigma_{k}=0.5\left(\left(\sigma_{k}\right)_{x}+\left(\sigma_{k}\right)_{y}\right)$

For the $\mathrm{x}$ - and $\mathrm{y}$-direction, $\sigma$ is given by

$\left(\sigma_{k}\right)_{x}=\frac{\operatorname{abs}\left(\mathcal{D}^{4}(\rho)_{k}\right)}{r_{g}+r_{k}}$

With $\mathcal{D}^{4}$ being the curvature of the curvature. In one dimension this quantity is defined as

$\mathcal{D}^{4}(\rho)_{k}=\mathcal{D}_{k+1}^{2}+\mathcal{D}_{k-1}^{2}-2 \mathcal{D}_{k}^{2}$

and the curvature $\mathcal{D}^{2}$ is given by

$\mathcal{D}_{k}^{2}(\rho)=\rho_{k+1}+\rho_{k-1}-2 \rho_{k}$

In the two dimensional case $\mathcal{D}$ is determined as the average scale in each of the two directions. The local range of $\rho$ defines the normalization, called $r_{k}$, given by

$$
\begin{aligned}
r_{k} & =\max \left[\rho_{l}, l=k-4, k+4\right] \\
& -\min \left[\rho_{l}, l=k-4, k+4\right]
\end{aligned}
$$


Ignoring truncation errors $r_{g}$ is proportional to the value $\mathcal{D}^{4}$ would take for a wave with wave number 1 . The value of $r_{g}$ is defined globally and used to avoid division by zero.

$r_{g}=\frac{\max \left(\rho_{l}, l=1, K\right)-\min \left(\rho_{l}, l=1, K\right)}{K^{4}}$

The target values, used for the filtering, are calculated from

$\rho_{T}=\max \left(\min \left(\rho_{A}, \rho_{\max }\right), \rho_{\min }\right)$

Details of the re-organisation of mass can be found in Kaas and Nielsen (2010). In brief, it consists of a small series of local mass-redistributions which gradually brings the mass conserved forecast closer to the target values. It can be thought of as a type of nudging under the strong constraint of local mass conservation.

\subsubsection{Trajectories}

Calculated trajectories need information about the wind in the current and the past time steps and use this information to extrapolate the wind velocity to the next time step. First, the "first guess" departure point is calculated. Using this point, the half time step forward trajectory is calculated. This is added to the half time step backward trajectory from the Eulerian arrival point. Going backwards along the resulting trajectory gives a new point, which is called the first iterated departure point. From this new point, a new half time step trajectory can be calculated, and so on. This iteration process can be repeated any number of times, but due to limitations in computer time and the fast convergence towards the "true" solution in the test cases considered here, the iteration is performed only twice in the present work.

The description of the calculation of trajectories follows that of Kaas (2008), bold italic font indicate vectors. The trajectory from the departure point, $\boldsymbol{r}_{\star}^{n}$, to the midpoint, $\boldsymbol{r}_{\star / 2}^{n+1 / 2}$, is calculated using Taylor series expansion:

$\boldsymbol{r}_{\star / 2}^{n+1 / 2}=\boldsymbol{r}_{\star}^{n}+\boldsymbol{C}_{1}$

where

$\boldsymbol{C}_{1}=\frac{\Delta t}{2} \boldsymbol{v}_{\star}^{n}+\sum_{m=1}^{M-1} \frac{1}{(m+1) !}\left(\frac{\Delta t}{2}\right)^{m+1}\left(\frac{d^{m} \boldsymbol{v}}{d t^{m}}\right)_{\star}^{n}$

In this work, $M=2$ is used; this includes acceleration. The term in the last parenthesis $\frac{d^{m} v}{d t^{m}}$ is approximated by $\frac{d v}{d t} \approx$ $\boldsymbol{v} \nabla \boldsymbol{v}$. This is done by not considering the Eulerian velocity change during the time step. For treatment of higher order derivatives the reader is referred to Kaas (2008).

The other piece of the trajectory is calculated in a similar way, now using extrapolated wind velocities rather than the velocity from the previous time step, $\tilde{\boldsymbol{v}}^{n+1}=2 \cdot \boldsymbol{v}^{n}-\boldsymbol{v}^{n-1}$. The last contribution to the trajectory is given as $\boldsymbol{r}_{\star / 2}^{n+1 / 2}=\boldsymbol{r}_{\star}^{n+1}+\boldsymbol{C}_{2}$

with

$$
\begin{aligned}
\boldsymbol{C}_{2} & =\frac{\Delta t}{2} \tilde{\boldsymbol{v}}^{n+1} \\
& -\sum_{m=1}^{M-1} \frac{1}{(m+1) !}\left(-\frac{\Delta t}{2}\right)^{m+1} \frac{d^{m}}{d t^{m}}\left(\tilde{\boldsymbol{v}}^{n+1}\right)
\end{aligned}
$$

Combining the above gives

$\boldsymbol{r}_{\star}^{n}=\boldsymbol{r}^{n+1}-\left(\boldsymbol{C}_{1}+\boldsymbol{C}_{2}\right)$

To achieve a higher accuracy $\boldsymbol{C}_{1}$ is iterated as mentioned above.

\subsection{Advection algorithm in DEHM}

The advection scheme used in DEHM to solve the twodimensional advection equation is the Accurate Space Derivatives (ASD) and, in addition, the Forester and Bartnicky filters are used to prevent the numerical oscillations and negative values that can occur when using a high order scheme. ASD is a pseudo-spectral method. The vertical advection is solved using a faster, less accurate finite-element method, Frohn et al. (2002). The temporal integration is performed using a third-order Taylor series method.

\subsubsection{Accurate space derivatives}

Accurate Space Derivatives use Fourier transforms to solve the two-dimensional advection. The change of the mixing ratio in one-dimension can be expressed as:

$c(x)=P(x)+F(x), \quad x \in[0, \ldots,(N-1) \cdot \Delta x]$

with

$$
\begin{aligned}
P(x) & =\left(\frac{c\left(x_{1}\right)-c\left(x_{N}\right)}{2}\right) \cos (\kappa \cdot x) \\
& +\left(\frac{c\left(x_{1}\right)-c\left(x_{N}\right)}{2}\right)
\end{aligned}
$$

The term $P(x)$ imposes boundary conditions on the system. For periodic boundary conditions $P(x)$ can be omitted, also, it is seen that for $c\left(x_{1}\right)=c\left(x_{N}\right) \Rightarrow P(x)=0$ for all $x$. The second term, $F(x)$, is the Fourier components given as

$F(x)=\sum_{n}\left(a_{n} \cos (2 \cdot n \cdot \kappa \cdot x)+b_{n} \sin (2 \cdot n \cdot \kappa \cdot x)\right)$

where $a_{n}$ and $b_{n}$ are the Fourier coefficients. $\kappa$ is

$\kappa=\frac{\pi}{N \cdot \Delta x}$

Taylor series expansion is used to perform the integration in time of the three-dimensional advection. In DEHM the 
Taylor series is expanded to third order:

$$
\begin{aligned}
c(t+\Delta t) \simeq & \sum_{n=0}^{3} \frac{1}{n !} \frac{\partial^{n} c}{\partial t^{n}} \cdot(\Delta t)^{n} \\
& =c(t)+c^{\prime}(t) \Delta t+\frac{1}{2} c^{\prime \prime}(t)(\Delta t)^{2}+\frac{1}{6} c^{\prime \prime \prime}(t)(\Delta t)^{3}
\end{aligned}
$$

The calculation of the derivatives, $c^{\prime}(t), c^{\prime \prime}(t)$ and $c^{\prime \prime \prime}(t)$, is performed using spacial derivatives:

$$
\begin{aligned}
c^{\prime}(t) & =-u \frac{\partial c}{\partial x}-v \frac{\partial c}{\partial y} \\
& =\bar{V} \cdot \bar{\nabla} c \\
c^{\prime \prime}(t) & =\bar{V} \cdot \bar{\nabla} c^{\prime} \\
c^{\prime \prime \prime}(t) & =\bar{V} \cdot \bar{\nabla} c^{\prime \prime}
\end{aligned}
$$

The analytical solution to $c^{\prime}(t)$, in one dimension, is given by:

$$
\begin{aligned}
c^{\prime}(x) & =P^{\prime}(x)+F^{\prime}(x) \\
c^{\prime}(x) & =-\kappa\left(\frac{c\left(x_{1}\right)-c\left(x_{N}\right)}{2}\right) \sin (\kappa \cdot x) \\
& +\sum_{n}\left(-2 \cdot n \cdot \kappa \cdot a_{n} \sin (2 \cdot n \cdot \kappa \cdot x)\right. \\
& \left.+2 \cdot n \cdot \kappa \cdot b_{n} \cos (2 \cdot n \cdot \kappa \cdot x)\right)
\end{aligned}
$$

Using an advection scheme with this high order of accuracy introduces a significant amount of numerical noise when considering steep gradients. Also overshoots and unphysical negative values might occur. To solve these problems, filters are introduced, in DEHM the Forester filter, see Forester (1977), and the Bartnicki filter, see Bartnicki (1989), are used. In a test performed by Dabdub and Seinfeld (1994), ASD and the Forester filter combined showed to be the best performing of the tested advection schemes. The Bartnicki filter removes negative values by redistribution of mass.

\subsubsection{The Forester filter}

The Forester filter works by adding dispersion to smooth the field where the peak values are present. On an iterative scheme the filter can be expressed as follows:

$$
\begin{aligned}
c_{i}^{k+1} & =c_{i}^{k}+\frac{\mu}{2}\left[\left(c_{i+1}-c_{i}\right)\left(\delta_{i}+\delta_{i+1}\right)\right. \\
& \left.-\left(c_{i}-c_{i-1}\right)\left(\delta_{i}+\delta_{i-1}\right)\right]^{k},
\end{aligned}
$$

where $k$ is the number of times the filter has been iterated. The four parameters $n, m, k$, and $\mu$ are specific for every implementation of the filter and must be determined empirically, $\delta$ is calculated using $n$ and $m$ and is either 0 or 1 depending of whether the filter is active or not. $k$ is the number of iterations the filter performs, $\mu$ is the diffusion coefficient which is dimensionless and dependent on, $C$, the Courant number. The noise wavelength is determined by $n$ and $m$. Local extrema are separated by $2 n$ using the local diffusion. The filter diffuses overshoots but does not prevent negative values, but reduces them. The filter is locally mass conserving.

\subsection{Test cases}

To test the different methods presented here the two classical tests, the slotted cylinder, see e.g. Zerroukat et al. (2002), and rotating cone by Molenkamp (1968) and Crowley (1968) are used. The rotation tests are applied both with and without chemistry. The tests have been modified to better test relevant properties of the schemes. For both test cases the object is rotated only one rotation, which for the chemistry corresponds to one day in this setup. This is chosen in order for significant features from the advection not to be hidden by the chemistry. Also the tests are run with some different number of time steps per rotation and different number of grid points; this is described in Sect. 5 .

When testing advection schemes combined with chemistry it is not certain that the results will be the same as when advection and chemistry are tested individually, Brandt et al. (1996b). For pure advection tests, the solution is well known. Also, for chemistry, the "real" solution can be found using the box model method, see Hov et al. (1989). Due to the non-linearity in the chemistry, the combined solution does not always perform as well as the individual results. Because of the above, in addition to showing the combined results including both advection and chemistry, figures showing the initial condition and the individual results for chemistry and pure advection are included for comparison.

The mathematical description of the tests can be found in e.g. Zerroukat et al. (2002) and Molenkamp (1968) or Crowley (1968).

\section{Algorithms}

In this section the different combinations of methods tested in this study are described along with the different resolutions used.

\subsection{Schemes}

In this work ten different methods are tested and compared. Two are based on the ASD method and the remaining eight methods are semi-Lagrangian schemes. Below is a list with description of every method. Section 4 describes the basic methods more thoroughly.

$A S D$ - Accurate Space Derivatives including the Bartnicki filter. This method is described in Sect. 4.4.1. This method is globally mass conserving and has no negative values.

$A S D$ w. filter - Accurate Space Derivatives with filter is the method described above combined with the Forester filter (see Sect. 4.4.2). This method is globally mass conserving and has no negative values.

$S L$ - semi-Lagrangian bi-cubic interpolation described in Sect. 4.3.2 
LMCSL - The Locally Mass Conserving semiLagrangian advection scheme is a combination of the classical semi-Lagrangian bi-cubic interpolation (SL) and the modified interpolation weights by Kaas (2008), this method is described in Sect. 4.3.4. This scheme is locally mass conserving but not monotonic.

SL w. filter - Classical bi-cubic semi-Lagrangian interpolation with the monotonic and mass conserving filter by Kaas and Nielsen (2010), see description in Sect. 4.3.5. This scheme is not mass conserving but monotonic.

LMCSL w. filter - This scheme combines the two methods above (LMCSL and SL w. filter). The base is the classical bi-cubic semi-Lagrangian interpolation, to this the modified interpolation weights which imposes mass conservation and the locally mass conserving monotonic filter are added. This scheme is both locally mass conserving and monotonic.

cascade - semi-Lagrangian cubic cascade interpolation, see Sect. 4.3.3. This method is faster than the traditional SL scheme but requires more memory.

LMC cascade - Locally Mass Conserving cascade interpolation. Here the modified interpolation weights are added to the cascade interpolation. This scheme is locally mass conserving but not monotonic.

cascade w. filter-Cascade interpolation with the locally mass conserving monotonic filter. This scheme is not mass conserving but monotonic.

LMC cascade w. filter - As the LMCSL with filter but using cascade interpolation. This scheme is both locally mass conserving and monotonic.

\subsection{Resolution}

The five different resolutions, which will be presented in the results in Sect. 6 are as follows. The first figure in the abbreviations below refers to the time step, i.e. 10_1, means that the time step is ten times larger than that of the reference time step used in ASD. The second figure refers to the spatial resolution, where "1" means standard resolution of the reference and " 05 " means that the resolution is half the standard resolution, i.e. twice as many grid cells in each direction.

$10 \_1$ - This is the standard setup for the semiLagrangian approach, see Sect. 4.5. In this setup the maximal Courant number is $C=3.27$, the time step is $\Delta t=900 \mathrm{~s}$ and the number of grid cells are $n x=n y=$ 100.

$1 \_1-$ This is the resolution ASD use as standard. The maximal Courant number is $C=0.327$, the time step is $\Delta t=90 \mathrm{~s}$ and the number of grid cells are $n x=n y=$ 100.
$3 \_l-$ This resolution is used to test the semi-Lagrangian schemes with a Courant number close to but not equal one, $C=0.981$, the time step is $\Delta t=270 \mathrm{~s}$ and the number of grid cells are $n x=n y=100$.

1_05 - The fourth resolution used has a maximal Courant number of 0.654 in each direction, the time step is $\Delta t=90 \mathrm{~s}$ and has twice as many grid points in each direction as the three first resolutions, $n x=n y=200$.

05_05 - In the last setup the maximal Courant number is the same as in the second resolution, $C=0.327$, however the time step is only half of that from the second method $\Delta t=45 \mathrm{~s}$ and the number of grid points is the same as that of the fourth method, $n x=n y=200$.

The ASD scheme is run only with maximal Courant number $C=0.327$, which corresponds to resolution $1 \_1$ and 05_05.

All eight semi-Lagrangian methods are run with all five resolutions and the two test cases described in Sect. 4.5. They are run with both analytical and calculated trajectories as described in Sect. 4.3.6.

The chemistry contains reactions for 58 species as described in Sect. 3.4.2. Since it would be beyond the scope of this work to go into detail with all chemical species, $\mathrm{O}_{3}$ and $\mathrm{NO}_{2}$ have been chosen for thorough investigation. $\mathrm{O}_{3}$ and $\mathrm{NO}_{2}$ describe both the evolution of species with an initial concentration different from the background concentration $\left(\mathrm{NO}_{2}\right)$ and a concentration changing from pure background to a higher and lower concentration $\left(\mathrm{O}_{3}\right)$.

\section{Results}

A systematic comparison of all the methods for two selected species $\left(\mathrm{NO}_{2}\right.$ and $\left.\mathrm{O}_{3}\right)$ using urban chemistry conditions is presented in Sect. 6.1. Only the rotating cone described in Sect. 4.5 has been used since the slope of the cone reflects what typically could occur in the real atmosphere. Each figure showing $\mathrm{NO}_{2}$ contains four plots, from top left to bottom right, the initial condition, pure advection, only chemistry, and last, advection and chemistry combined. For $\mathrm{O}_{3}$, the initial condition is pure background concentration which makes pure advection and initial condition unimportant, therefore, for $\mathrm{O}_{3}$ just two plots, chemistry and advection and chemistry combined, are shown.

In the ranking, see Sect. 6.3, of the different methods the ASD scheme without the Forester filter has been included, however, in the figures below no plots of ASD without filter occur and ASD refers to the ASD scheme with both the Forester filter and the Bartnicki filter.

In Sect. 6.2 the slotted cylinder is used to test the methods on a very steep gradient. It is very difficult for numerical methods to model this steep slope without generating numerical noise known as the Gibbs phenomenon. In Sect. 6.2 the 

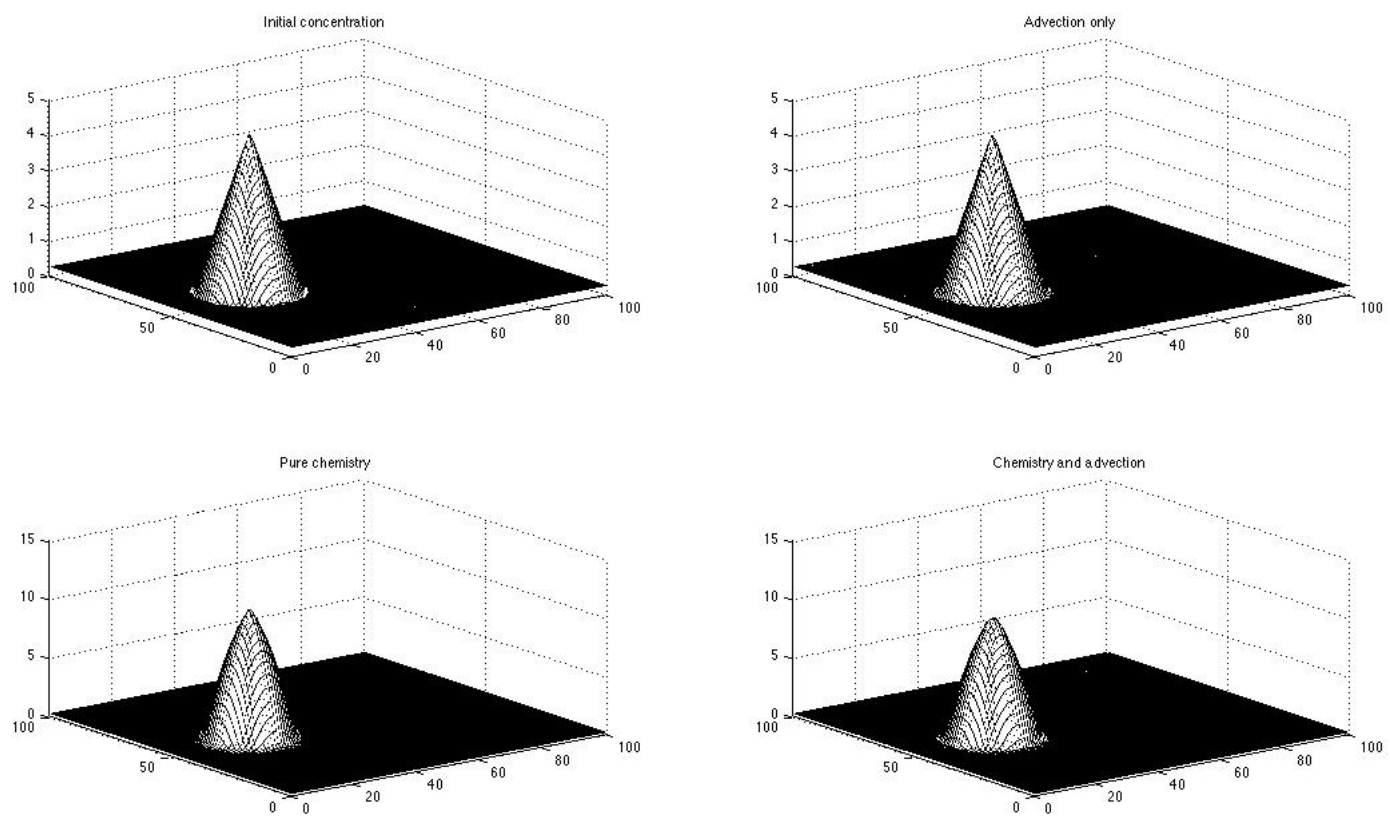

Fig. 1. Rotation test for ASD using the rotating cone for $\mathrm{NO}_{2}$ with urban conditions and resolution $1 \_1$, with $\Delta t=90 \mathrm{~s}, \Delta x=1.0$, and $C=0.327$. The results are given for the initial condition (top left) and model runs with pure advection (top right), pure chemistry (bottom left), and combined chemistry and advection (bottom right).

The minimum and maximum values for the respective plots are:

Top left: $\min =0.2406, \max =4.729$. Top right: $\min =0.2221, \max =4.683$.

Bottom left: $\min =0.1317, \max =11.22$. Bottom right: $\min =0.106, \max =10.44$.

results for the two best performing semi-Lagrangian schemes are compared to ASD.

Error measures and ranking are shown and explained in Sect. 6.3. Finally a discussion of the computational efficiency of the various methods is found in Sect. 6.4.

\subsection{Comparison of results for $\mathrm{NO}_{2}$ and $\mathrm{O}_{3}$ using the rotating cone}

For an ideal scheme, the results from the rotation test after a full rotation using pure advection should be impossible to tell apart from the initial condition. Similarly, for pure chemistry compared to combined chemistry and advection. However, when including non-linear chemistry this constraint is even more severe than when considering a passive tracer.

\subsubsection{Results for $\mathrm{NO}_{2}$ using the rotating cone}

In the following plots the results obtained using no advection and pure chemistry, are considered the "reference" solutions to the results including advection alone, and advection together with chemistry, respectively. This section considers results for the chemical component $\mathrm{NO}_{2} . \mathrm{NO}_{2}$ is present from the beginning of the test and therefore shows both advection and change in concentration due to chemistry.

The top left plot in the Figs. 1-3 shows the initial condition, that is the concentration of $\mathrm{NO}_{2}$ shaped as a cone, before both advection or chemistry, at time $t=0$. This is to be compared to the top right plot showing the performance of the considered advection scheme, still without active chemistry, this corresponds to passive tracer advection. In the figures the vertical axes on the top plots are in the interval 0-5 and the bottom plots, in the interval 0-15.

The bottom row includes active chemistry. The bottom left plot shows the concentration of the field when only chemistry is active, this is the ideal solution for an advection scheme with active chemistry. The bottom right figure shows the performance of the advection scheme including active chemistry. The ranking of the schemes can be found in Table 3 .

When considering only the two left plots, initial condition and pure chemistry, the effect of active chemistry after $24 \mathrm{~h}$ is seen. The concentration increases from a maximum of 4.729 to 11.22 and the background concentration changes from 0.2406 to 0.1317 . It would be beyond the scope of this work to go into detail with the chemical reactions causing this evolution. It is noted that the shape of the rotating cone is somewhat rounded by the chemical reactions alone.

\subsubsection{Results for $\mathrm{NO}_{2}$ using the ASD approach}

In Fig. 1 the advection scheme from DEHM, ASD, is tested. The results considering only pure advection show a very well preserved cone, the extreme values are slightly lower than 

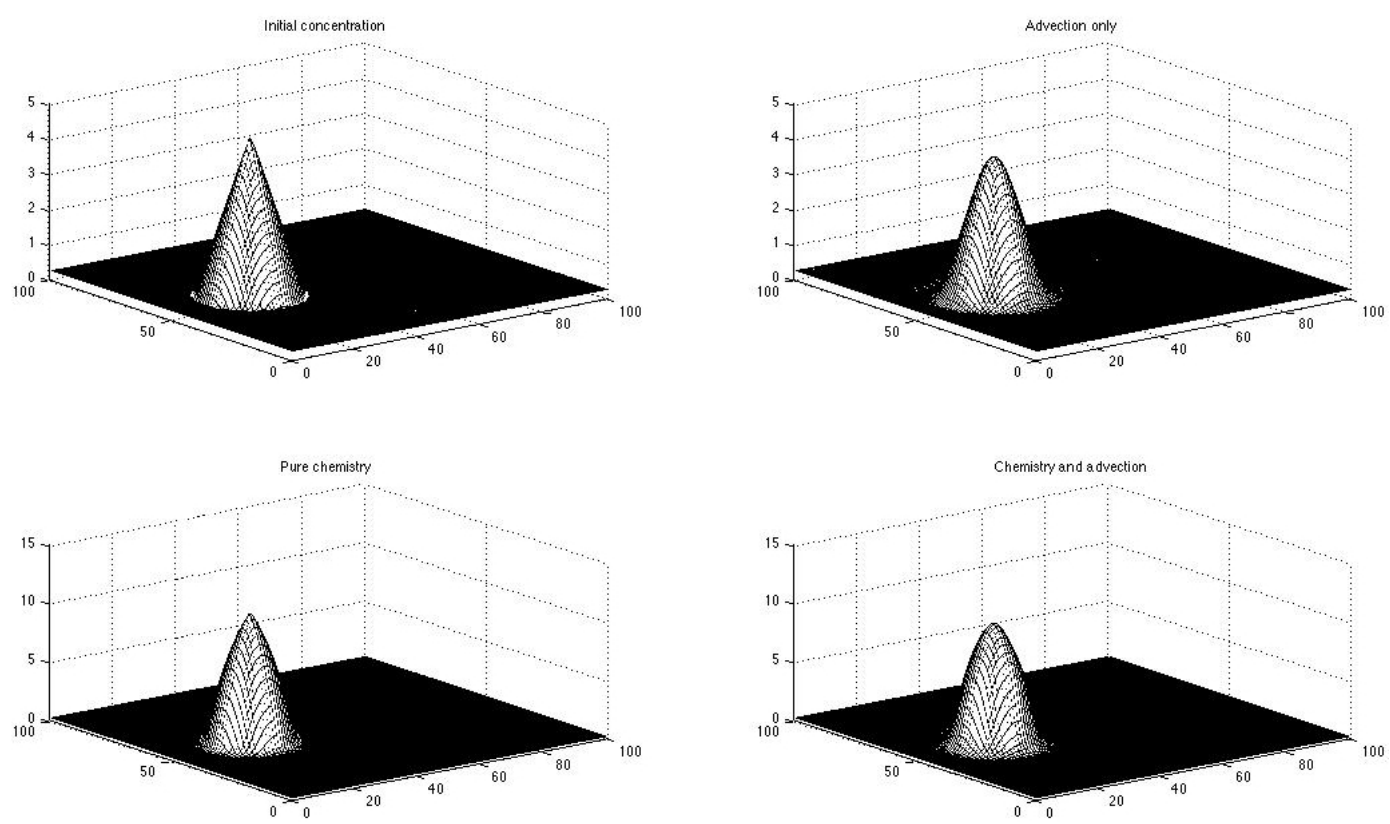

Fig. 2. Rotation test for $\mathrm{LMC}$ cascade using the rotating cone for $\mathrm{NO}_{2}$ with urban conditions and resolution $1 \_1$, with $\Delta t=90 \mathrm{~s}, \Delta x=1.0$, and $C=0.327$. The results are given for the initial condition and model runs with pure advection (top right), pure chemistry (bottom left), and combined chemistry and advection (bottom right).

The minimum and maximum values for the respective plots are:

Top left: $\min =0.2406, \max =4.729$. Top right: $\min =0.1929, \max =4.2$.

Bottom left: $\min =0.1317, \max =11.22$. Bottom right: $\min =0.0397, \max =10.31$.
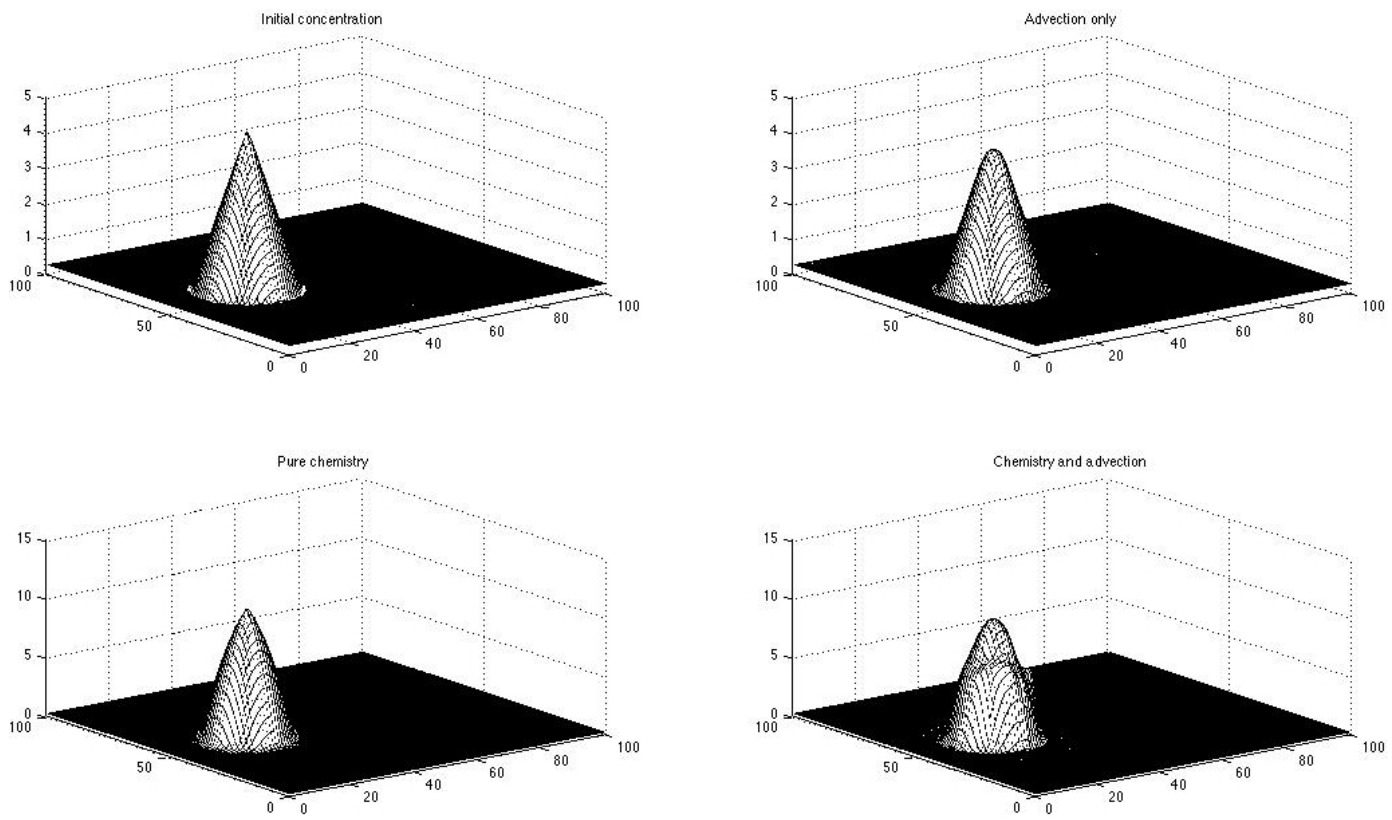

Fig. 3. Rotation test for LMCSL with filter using the rotating cone for $\mathrm{NO}_{2}$ with urban conditions and resolution $1 \_1$, with $\Delta t=90 \mathrm{~s}$, $\Delta x=1.0$, and $C=0.327$. The results are given for the initial condition (top left) and model runs with pure advection (top right), pure chemistry (bottom left), and combined chemistry and advection (bottom right).

The minimum and maximum values for the respective plots are:

Top left: $\min =0.2406, \max =4.729$. Top right: $\min =0.2406, \max =4.221$.

Bottom left: $\min =0.1317, \max =11.22$. Bottom right: $\min =0.0295, \max =10.33$. 
those of the initial condition, 0.2221 and 4.683 compared to 0.2406 and 4.729 .

When including active chemistry the result is similar to the above, again the extrema are a little different from those of the pure chemistry, 0.106 and 10.44 compared to 0.1317 and 11.22. This is an acceptable change.

The ASD preserves the shape of the cone very well, when only considering advection even the top of the cone stays "pointy" as in the initial condition. When active chemistry and advection is combined the top of the cone is slightly rounded as compared to that of both advection alone and pure chemistry. The non-linearities of the chemistry cause this change.

\subsubsection{Results for $\mathrm{NO}_{2}$ using the semi-Lagrangian cascade approach}

The result of combining the semi-Lagrangian cascade scheme with modified interpolation weights, LMC cascade, is shown in Fig. 2. When comparing the top plots it is easily seen that the scheme smoothes the solution, in particular the top of the cone is rounded somewhat. The extreme values also show this, the maximum of the advected cone is 4.2 compared to 4.729 for the initial condition. When the chemistry is included, the extreme values are 0.0397 and 10.31 compared to 0.1317 and 11.22 and as before the top of the cone is smoothed. The modified interpolation weights make the scheme mass conserving, but do not alter the extrema.

\subsubsection{Results for $\mathrm{NO}_{2}$ using the filtered mass conserving semi-Lagrangian Approach}

The last figure with this resolution, Fig. 3, combines the three of the schemes considered, semi-Lagrangian interpolation, the modified interpolation weights and the locally mass conserving monotonic filter to one, namely LMCSL with filter. This combined scheme is both monotonic and mass conserving. The minimum value of the result of the pure advection is the same as the initial concentration, namely 0.2406 , the maximum value is 4.221 against 4.729 from the initial concentration. As for the scheme with chemistry the lowest and highest values are 0.0295 and 10.33 , respectively. The corresponding values for the reference solution are 0.1317 and 11.22. However, the shape of the cone seems broader than the reference. The most significant feature is a "bulk" on the cone one third of the cone height from the top, resulting from the filter and the non linear chemistry.

\subsubsection{Results for $\mathrm{NO}_{2}$ using the semi-Lagrangian cascade approach with resolution $3 \_1$}

Figure 4 shows the same chemical species as discussed above, namely $\mathrm{NO}_{2}$, and the same test, the rotating cone, however, using resolution 3_1. By comparing the previous three figures, the bottom right plot, it can be seen that the top of the cone is slightly rounded, like the other semiLagrangian schemes, however, the maximum value is higher than even the result produced by the ASD scheme. The bulk occurring on the plots from the filtered semi-Lagrangian schemes using resolution 1_1 is no longer there on this figure. The minimum value of the bottom right plot is closer to the value for pure chemistry than for the other semi-Lagrangian schemes but still worse than the result from the ASD scheme. When considering pure advection, the minimum values are the same for all semi-Lagrangian schemes, namely the same as the initial condition. The maximum value is again closer to the initial condition than the semi-Lagrangian schemes using resolution 1_1, but not as close as the maximum value for the ASD scheme.

\subsubsection{Comparison of results for $\mathrm{NO}_{2}$}

The results above show that the ASD scheme is very good at preserving shape when considering the rotating cone. However, when chemistry is included, the non-filtered results of the semi-Lagrangian schemes and those with a long time step perform almost as well.

When considering pure advection the filtered solution of the semi-Lagrangian schemes performs very well. The bulk occurring when the scheme is combined with the filter might result from the non-linearities in the chemistry or the filter might be trying "too hard" to keep the gradients steep.

When adding the modified interpolation weights to a scheme, it becomes mass conserving. When applying the locally mass conserving monotonic filter to a scheme which is not mass conserving, the scheme becomes monotonic, but not mass conserving, because the filter only conserves the mass of the result from the advection scheme, which is not mass conserving.

\subsubsection{Results for $\mathrm{O}_{3}$ using the rotating cone}

The initial $\mathrm{O}_{3}$ concentration is prescribed as a constant background concentration. It is challenging for advection schemes to model the changing concentration correctly when it occurs due to pure chemical reactions.

In Figs. 5-7 the vertical axis is in the interval 40-80. Each figure shows a plot of pure chemistry and a plot of advection and chemistry. The reason that neither the initial condition nor pure advection are shown is that the concentration of the two is constant throughout the grid.

The pure chemistry plot is considered the reference solution, it has a minimum concentration of 48.55 and a maximum concentration of 64.58 , the shape of the concentration looks like a cone where the center bulks downward.

\subsubsection{Results for $\mathrm{O}_{3}$ using the ASD approach}

Figure 5 shows the result obtained using ASD. The shape of the cone is comparatively well preserved, with only a small undershoot, minimum value $=48.18$ compared to 48.55 . The 

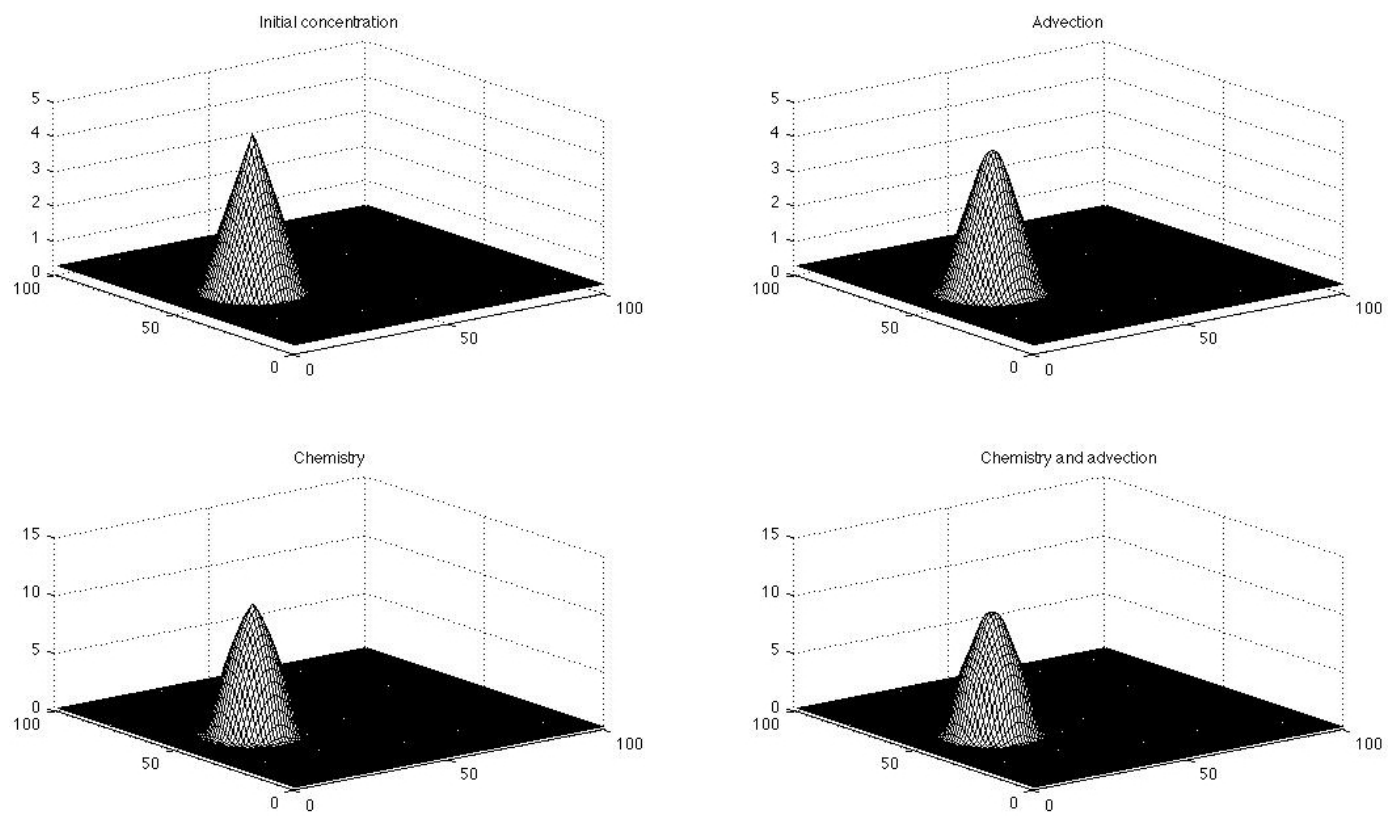

Fig. 4. Rotation test for filtered $\mathrm{LMC}$ cascade using the rotating cone for $\mathrm{NO}_{2}$ with urban conditions and resolution 3_1, with $\Delta t=270 \mathrm{~s}$, $\Delta x=1.0$, and $C=0.981$. The results are given for the initial condition (top left) and model runs with pure advection (top right), pure chemistry (bottom left), and combined chemistry and advection (bottom right).

The minimum and maximum values for the respective plots are:

Top left: $\min =0.2406, \max =4.729$. Top right: $\min =0.2406, \max =4.263$.

Bottom left: $\min =0.1328, \max =11.31$. Bottom right: $\min =0.0838, \max =10.56$.
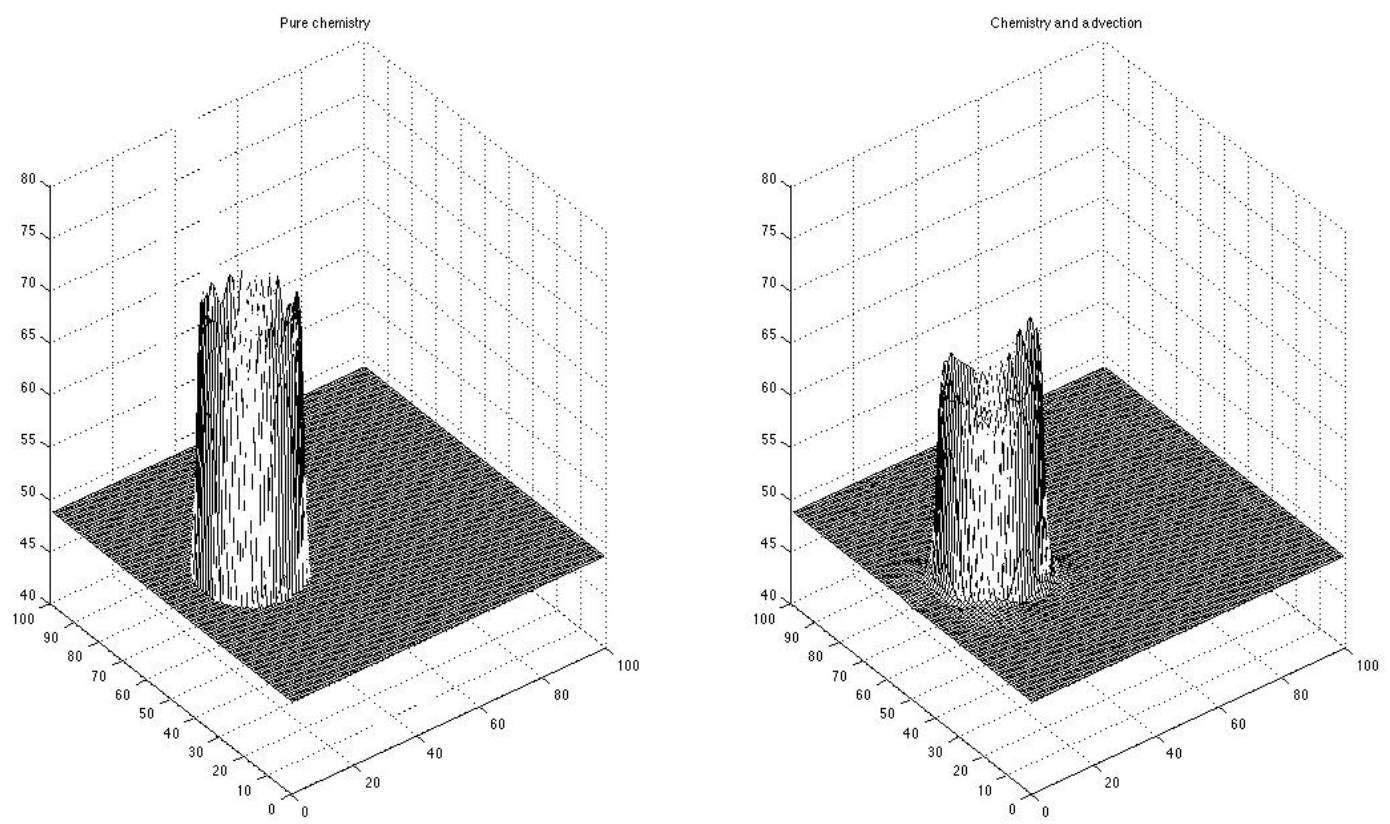

Fig. 5. Rotation test for ASD using the rotating cone for $\mathrm{O}_{3}$ with urban conditions and resolution $1 \_1$, with $\Delta t=90 \mathrm{~s}$, $\Delta x=1.0$, and $C=0.327$. The results are given for pure chemistry (left) and combined chemistry and advection (right).

The minimum and maximum values for the respective plots are:

Left: minimum $=48.55$, aximum $=64.58$.

Right: minimum $=48.18$, maximum $=71.23$. 

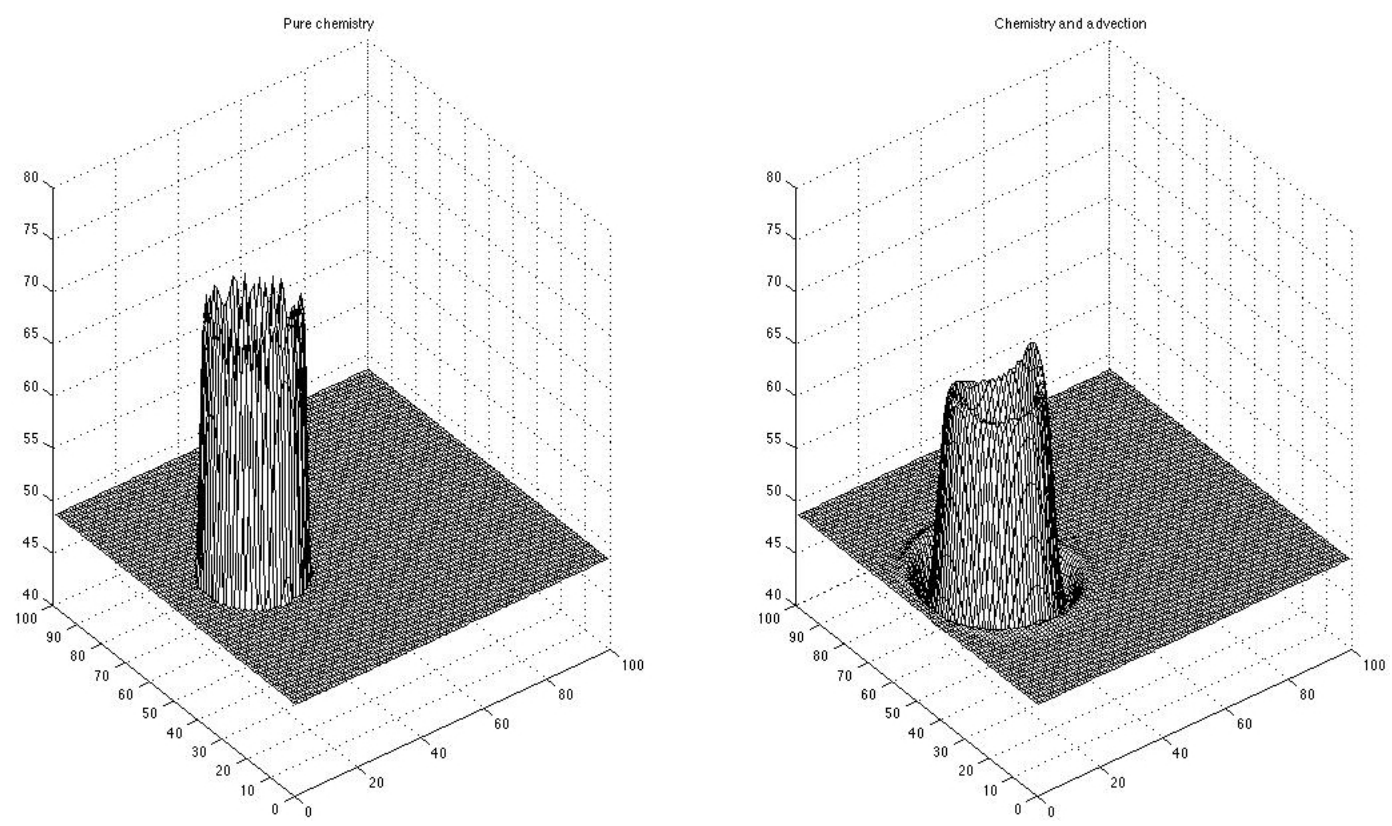

Fig. 6. Rotation test for $\mathrm{LMC}$ cascade using the rotating cone for $\mathrm{O}_{3}$ with urban conditions and resolution $1 \_1$, with $\Delta t=90 \mathrm{~s}, \Delta x=1.0$, and $C=0.327$. The results are given for pure chemistry (left) and combined chemistry and advection (right).

The minimum and maximum values for the respective plots are:

Left: minimum $=48.55$, maximum $=64.58$.

Right: minimum $=44.41$, maximum $=69.15$.
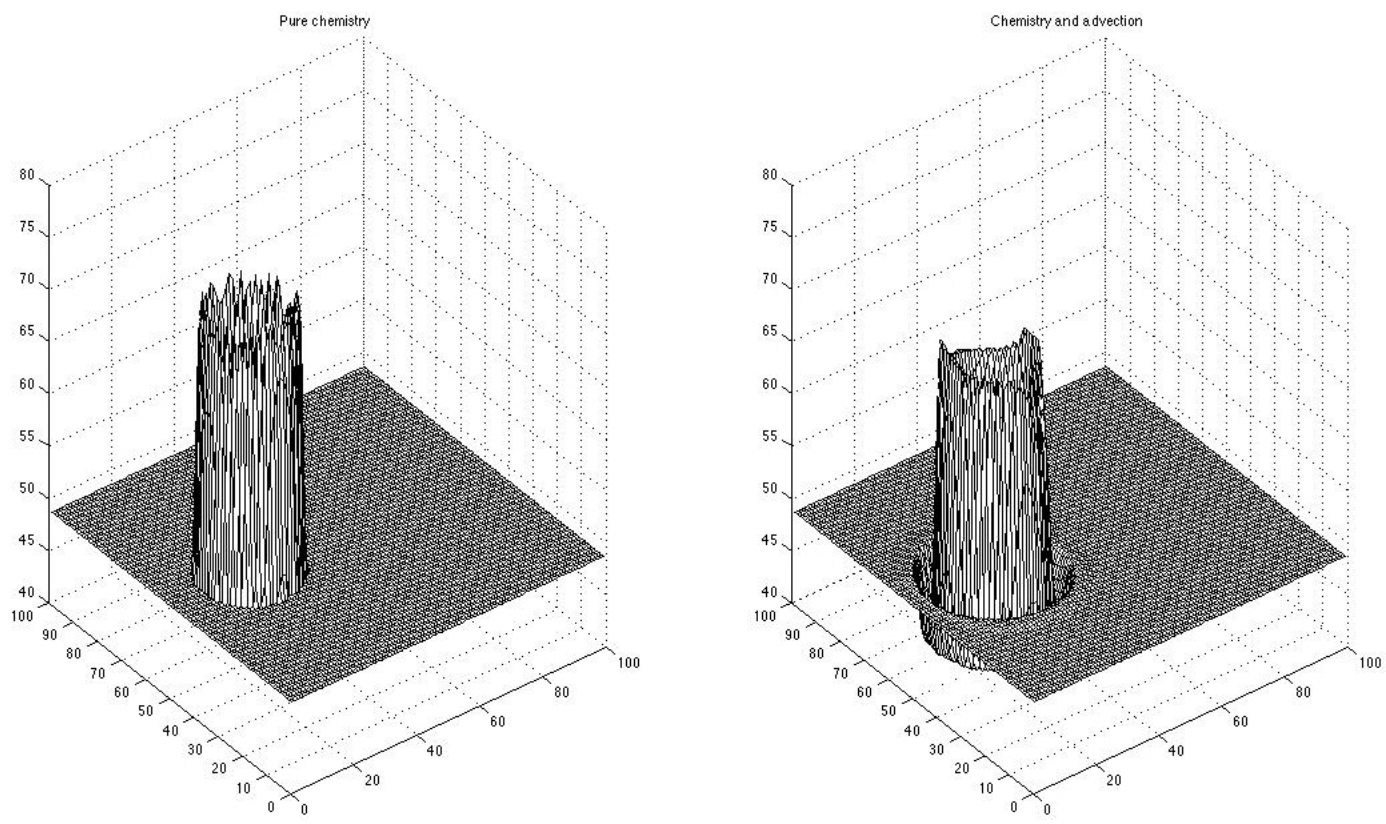

Fig. 7. Rotation test for LMCSL with filter using the rotating cone for $\mathrm{O}_{3}$ with urban conditions and resolution $1 \_1$, with $\Delta t=90 \mathrm{~s}, \Delta x=1.0$, and $C=0.327$. The results are given for pure chemistry (left) and combined chemistry and advection (right).

The minimum and maximum values for the respective plots are:

Left: minimum $=48.55$, maximum $=64.58$.

Right: minimum $=43.03$, maximum $=70.89$. 

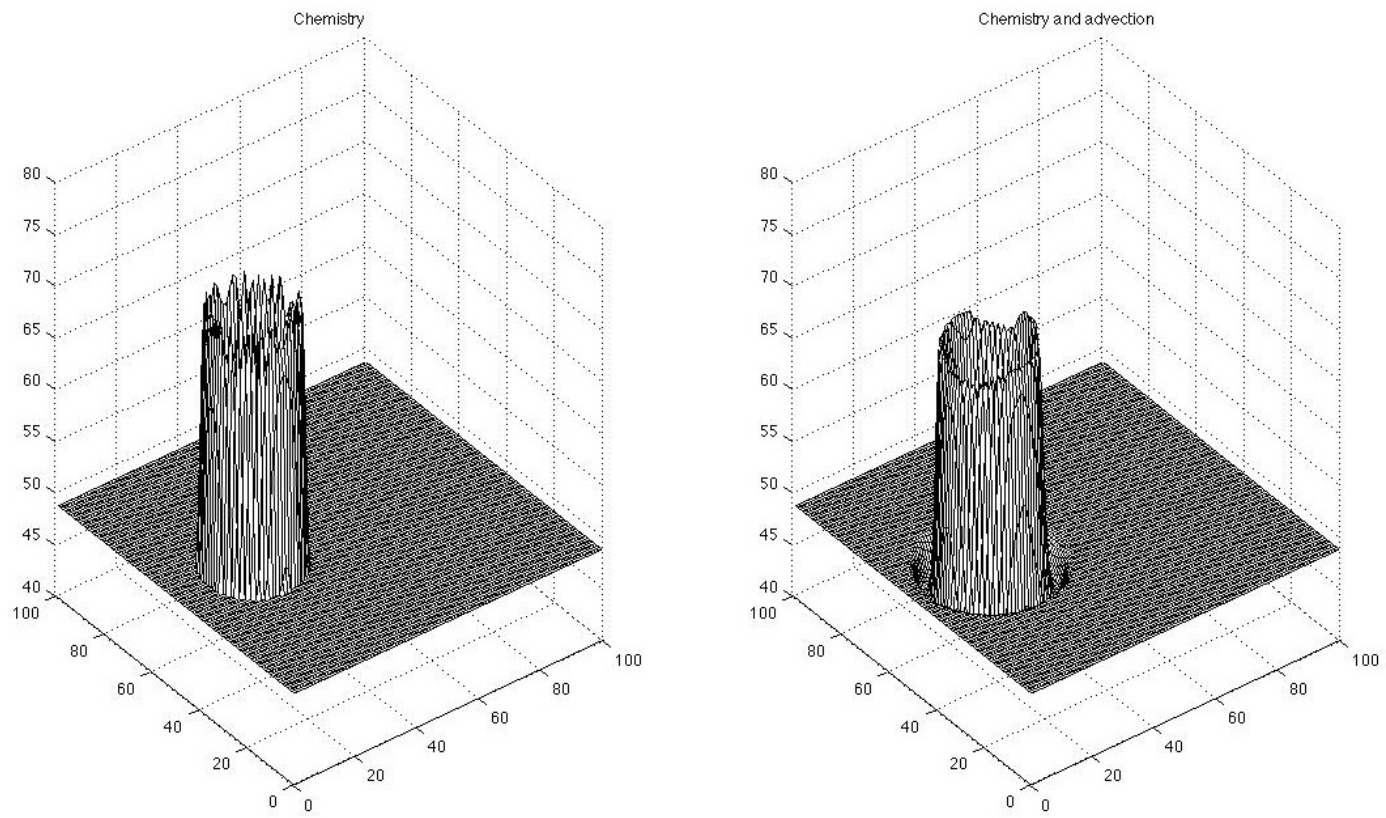

Fig. 8. Rotation test for LMC cascade with filter using the rotating cone for $\mathrm{O}_{3}$ with urban conditions and resolution 3_1, with $\Delta t=270 \mathrm{~s}$, $\Delta x=1.0$, and $C=0.981$. The results are given for pure chemistry (left) and combined chemistry and advection (right).

The minimum and maximum values for the respective plots are:

Left: minimum $=48.53$, maximum $=74.14$.

Right: minimum $=46$, maximum $=71.26$.

top of the concentration, however, generates a peak on one side of the cone with a maximum value of 71.23 compared to 64.58 .

\subsubsection{Results for $\mathrm{O}_{3}$ using the semi-Lagrangian cascade approach}

When considering Fig. 6, the scheme using the locally mass conserving cascade interpolation, it is seen that the scheme smoothes the solution. Also, a clear undershoot occurs, the minimum value of 44.41 compared to 48.55 confirms this. The overshoot generated is smaller than that for the ASD scheme, 69.15 compared to the reference solution with 64.58; this is still significant.

\subsubsection{Results for $\mathrm{O}_{3}$ using the semi-Lagrangian approach}

When adding the filter to the mass conservative semiLagrangian scheme, the gradient is steepened and the amplitude of the concentration increases. For Fig. 7, the extreme values are 43.03 and 70.89 , compared to 48.55 and 64.58 .

\subsubsection{Results for $\mathrm{O}_{3}$ using the semi-Lagrangian approach using resolution 3_1}

Figure 8 shows the combination of semi-Lagrangian cascade interpolation with modified interpolation weights and the locally mass conserving and monotonic filter using resolution 3_1. From the extreme values, it can be seen that some under and over shooting still occurs, however, the undershooting is significantly smaller than for the semiLagrangian schemes using resolution 1_1. The overshoot is comparable to that of the ASD scheme and therefore greater than those of the semi-Lagragian schemes. The peak in the concentration seems to be more evenly distributed than what other schemes present.

\subsubsection{Comparison of results for $\mathrm{O}_{3}$}

The ASD scheme generates a clear overshoot on one side of the cone. The semi-Lagrangian schemes also generate overshoots, however, significantly smaller. The semi-Lagrangian schemes generate severe undershoots and smooth the shape of the cone significantly. When a filter is added the shape is better preserved but both the undershoots and overshoots are enlarged.

\subsection{Selected results using the slotted cylinder}

In this section, selected results using the slotted cylinder test case are shown. For most of the plots the LMCSL scheme with the locally mass conserving monotonic filter has been chosen. The reason for choosing this scheme is that it gives the best scores for all tests when not considering ASD; this will be shown in Sect. 6.3. Results for the two 

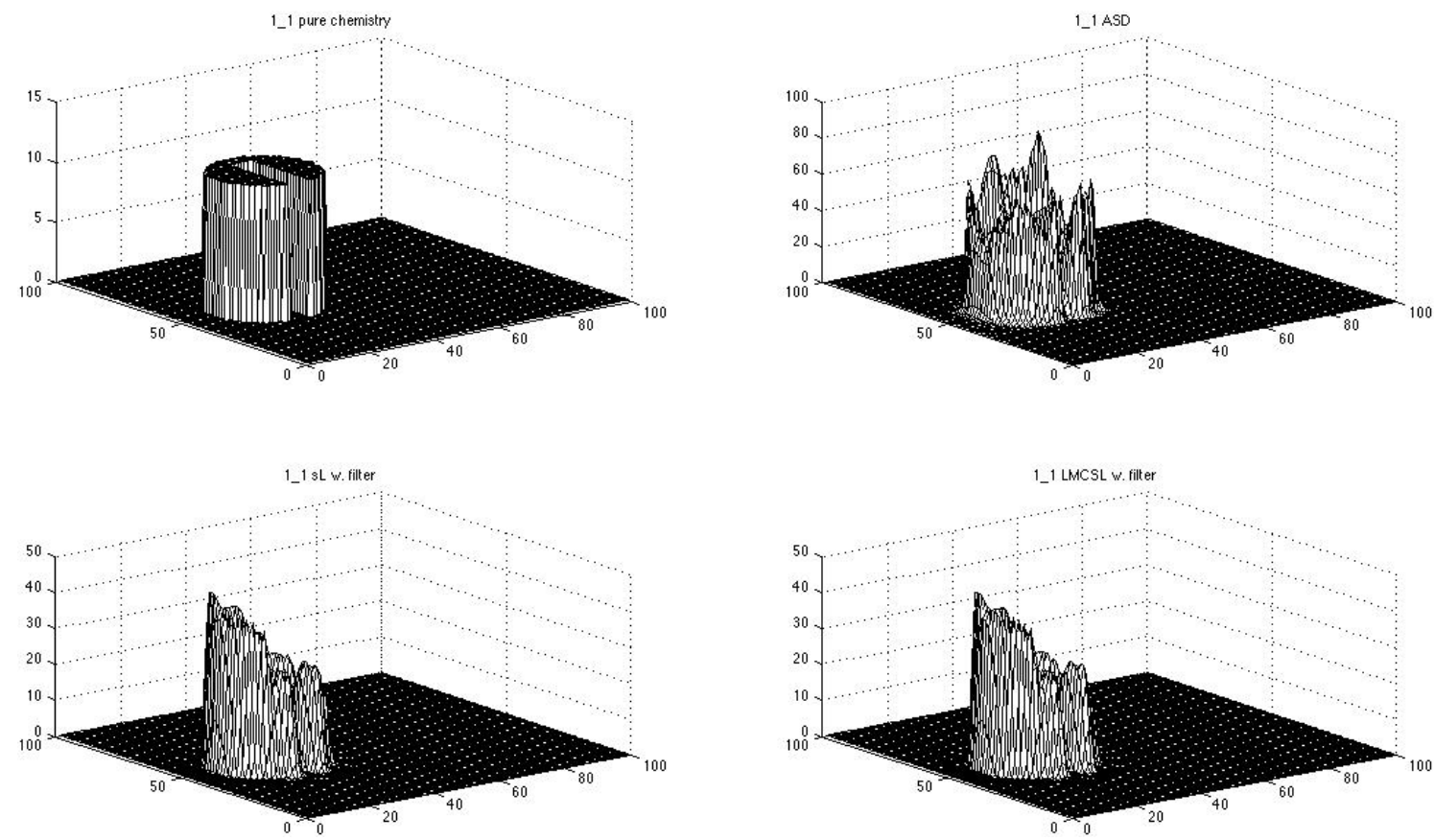

Fig. 9. Slotted cylinder, $\mathrm{NO}_{2}, \Delta t=90 \mathrm{~s}, \Delta x=1.0, C=0.327$ Rotation test with the slotted cylinder for $\mathrm{NO}_{2}$ using urban chemistry and resolution 1_1, with $\Delta t=90 \mathrm{~s}, \Delta x=1.0$, and $C=0.327$. The results are given for pure chemistry (top left) and chemistry and advection using ASD (top right), using semi-Lagrangian interpolation with filter (bottom left), and using LMCSL with filter (bottom right). Note, the vertical axis on the top left plot is the interval $0-15$, whereas the top right is in the interval $0-100$, and the two bottom plots are in the interval $0-50$.

The minimum and maximum values for the respective plots are:

Top left: $\min =0.1317, \max =11.36$. Top right: $\min =0.0016, \max =89.15$.

Bottom left: $\min =0.0056, \max =48.21$. Bottom right: $\min =0.005, \max =48.31$.

best performing SL schemes when considering urban chemistry and tested on the slotted cylinder with urban chemistry are chosen, see Table 4. Since the classical SL scheme combined with the filter is the best in three of the six tests it was decided that it should also be included when showing results in this section.

The plots in this subsection show (from upper left to lower right) pure chemistry, which is the reference solution, ASD, SL with filter, and LMCSL with filter.

Figure 9 shows the results obtained for $\mathrm{NO}_{2}$. The scales on the four plots vary, for the initial condition the minimum value is 0.1317 and the maximum value is 11.36. The ASD scheme generates very high peaks, and the lowest minimum value of the shown plots as well, minimum value $=0.0016$ and maximum value $=89.15$. Also, the semi-Lagrangian schemes give results different from the not advected solution, the minimum and maximum values for the classical scheme with filter are 0.0056 and 48.21 , respectively. For the mass conserving LMCSL scheme with filter, the values are only slightly different from those of the classical SL scheme with filter: the minimum is 0.005 and the maximum is 48.31 .
When considering $\mathrm{O}_{3}$, see Fig. 10, the same results as above can be seen. The ASD scheme is not performing as well as the semi-Lagrangian schemes; however, for $\mathrm{O}_{3}$ all schemes generate large undershoots. The reference solution has a minimum value of 48.55 and a maximum value of 60.05. The ASD schemes extreme values are 1.618 and 53.76, but as opposed to the reference solution, the peak of the concentration is "negative" relative to the background concentration. The semi-Lagrangian schemes again perform very similar. The minimum value is 15.41 for the non-mass conserving scheme and 15.32 for the LMCSL scheme, both with filter. The maximum value for both schemes is 75.91. The shape of the cylinder is altered significantly compared to the reference solution, but also very different from the solution obtained using ASD. The cylinder peaks both above and below the background concentration and the slot is somewhat preserved. 

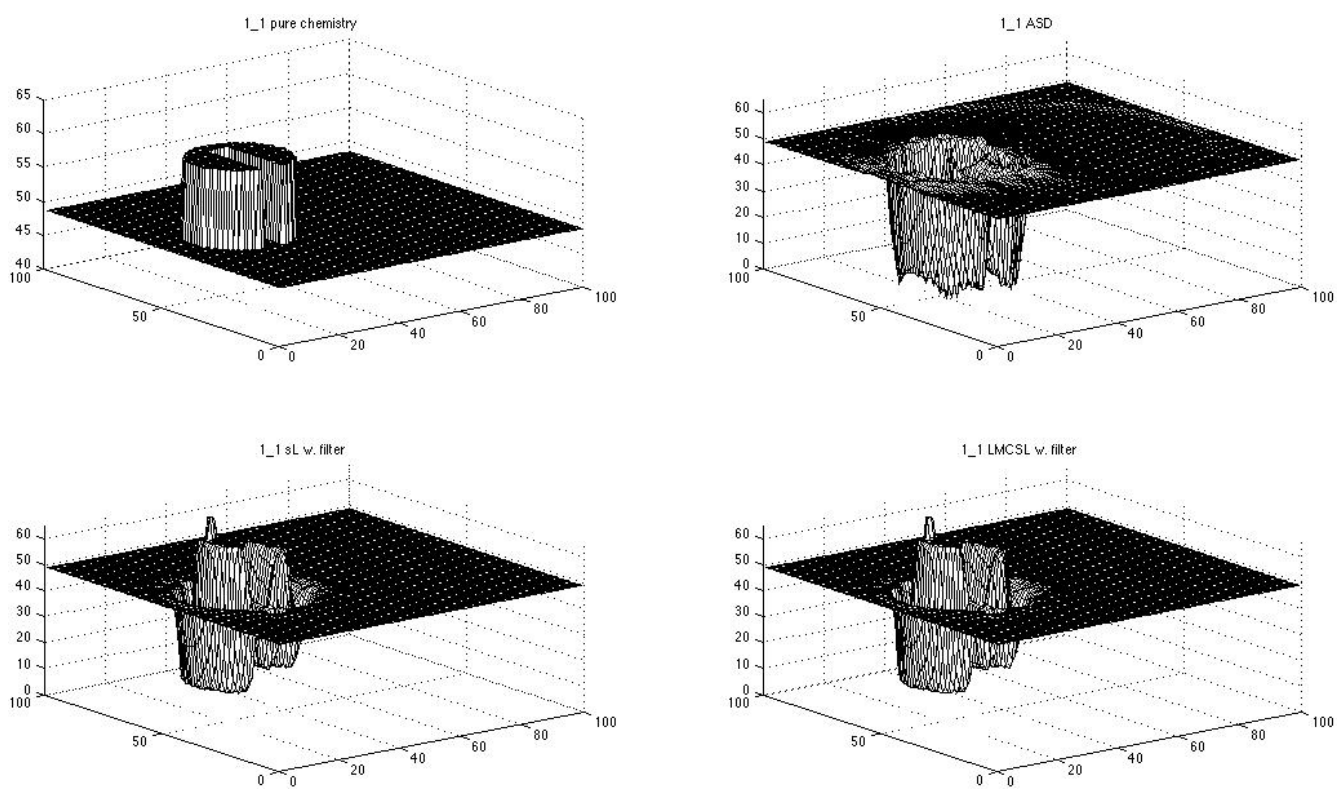

Fig. 10. Slotted cylinder, $\mathrm{O}_{3}, \Delta t=90 \mathrm{~s}, \Delta x=1.0, C=0.327$ Rotation test with the slotted cylinder for $\mathrm{O}_{3}$ using urban chemistry and resolution 1_1, with $\Delta t=90 \mathrm{~s}, \Delta x=1.0$, and $C=0.327$. The results are given for pure chemistry (top left) and chemistry and advection using ASD (top right), using semi-Lagrangian interpolation with filter (bottom left), and using LMCSL with filter (bottom right). Note, the vertical axis on the top left plot is the interval 40-65, whereas the other are in the interval 0-65.

The minimum and maximum values for the respective plots are:

Top left: $\min =48.55, \max =60.05$. Top right: $\min =1.618, \max =53.76$.

Bottom left: $\min =15.41, \max =75.91$. Bottom right: $\min =15.32, \max =75.91$.
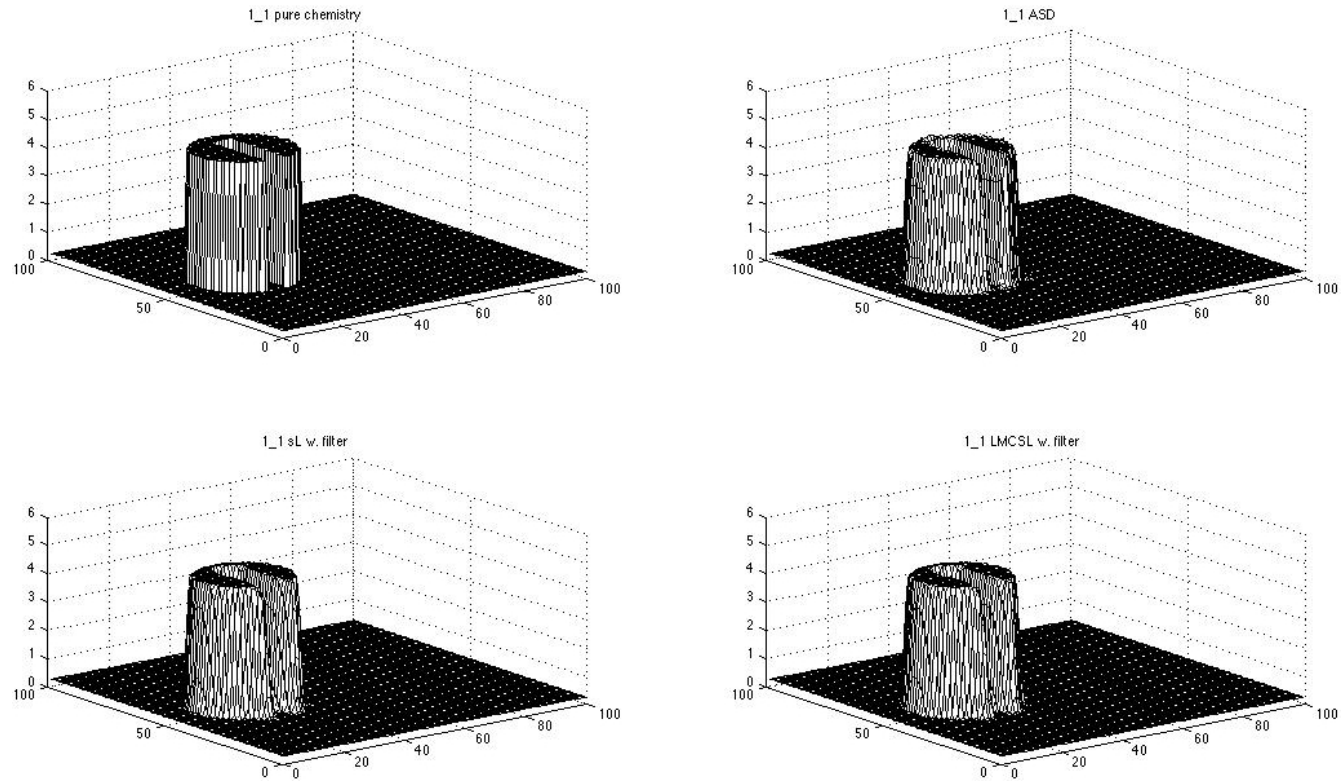

Fig. 11. Slotted cylinder, pure advection, $\Delta t=90 \mathrm{~s}, \Delta x=1.0, C=0.327$ Rotation test with the slotted cylinder for pure advection and resolution 1_1, with $\Delta t=90 \mathrm{~s}, \Delta x=1.0$, and $C=0.327$. The results are given for initial concentration (top left) and pure advection for ASD (top right), semi-Lagrangian interpolation with filter (bottom left), and LMCSL with filter (bottom right).

The minimum and maximum values for the respective plots are:

Top left: $\min =0.2406, \max =4.811$. Top right: $\min =0.0572, \max =5.022$.

Bottom left: $\min =0.2406, \max =4.811$. Bottom right: $\min =0.2406, \max =4.811$. 
Table 1. Ranking results for the rotating cone with rural chemistry. Showing rank of the ranked error measures, rank of error measure $l_{1}, l_{2}$, and $l_{\infty}$ followed by method, maximum Courant number, grid resolution, and time step.

\begin{tabular}{|c|c|c|c|c|c|c|c|}
\hline $\operatorname{rank(all)}$ & $\operatorname{rank}\left(l_{1}\right)$ & $\operatorname{rank}\left(l_{2}\right)$ & $\operatorname{rank}\left(l_{\infty}\right)$ & Method & $\mathrm{CFL}_{\max }$ & $\Delta x$ & $\Delta t$ \\
\hline 1.46 & 2.12 & 1.04 & 1.22 & ASD & 0.327 & 0.5 & 45 \\
\hline 1.67 & 1.25 & 1.96 & 1.79 & ASD w. filter & 0.327 & 0.5 & 45 \\
\hline 3.53 & 4.44 & 3.17 & 2.99 & ASD & 0.327 & 1.0 & 90 \\
\hline 3.85 & 3.25 & 4.18 & 4.13 & ASD w. filter & 0.327 & 1.0 & 90 \\
\hline 7.65 & 7.68 & 7.37 & 7.92 & LMCSL w. filter & 0.654 & 0.5 & 90 \\
\hline 7.77 & 7.61 & 7.56 & 8.13 & SL w. filter & 0.654 & 0.5 & 90 \\
\hline 8.73 & 8.61 & 8.70 & 8.88 & cascade w. filter & 0.654 & 0.5 & 90 \\
\hline 9.12 & 8.56 & 8.97 & 9.84 & LMCcascade w. filter & 0.654 & 0.5 & 90 \\
\hline 13.79 & 11.93 & 12.26 & 17.19 & LMCSL w. filter & 0.327 & 0.5 & 45 \\
\hline 14.69 & 12.10 & 13.11 & 18.84 & SL w. filter & 3.27 & 1.0 & 900 \\
\hline 15.44 & 13.42 & 14.18 & 18.73 & LMCcascade w. filter & 0.327 & 0.5 & 45 \\
\hline 15.72 & 14.24 & 13.49 & 19.43 & LMCSL w. filter & 3.27 & 1.0 & 900 \\
\hline 16.00 & 18.04 & 16.88 & 13.08 & SL & 0.654 & 0.5 & 90 \\
\hline 16.01 & 18.04 & 16.88 & 13.12 & LMCSL & 0.654 & 0.5 & 90 \\
\hline 16.02 & 18.04 & 16.93 & 13.08 & cascade & 0.654 & 0.5 & 90 \\
\hline 16.02 & 18.04 & 16.93 & 13.08 & LMCcascade & 0.654 & 0.5 & 90 \\
\hline 16.06 & 12.33 & 13.84 & 22.00 & cascade w. filter & 0.327 & 0.5 & 45 \\
\hline 16.43 & 13.48 & 15.18 & 20.62 & SL w. filter & 0.327 & 0.5 & 45 \\
\hline 18.10 & 17.24 & 20.69 & 16.37 & cascade w. filter & 3.27 & 1.0 & 900 \\
\hline 20.74 & 23.07 & 21.24 & 17.91 & cascade & 0.327 & 0.5 & 45 \\
\hline 20.74 & 23.07 & 21.24 & 17.91 & LMCcascade & 0.327 & 0.5 & 45 \\
\hline 20.74 & 23.07 & 21.24 & 17.91 & SL & 0.327 & 0.5 & 45 \\
\hline 20.74 & 23.07 & 21.24 & 17.91 & LMCSL & 0.327 & 0.5 & 45 \\
\hline 21.94 & 19.40 & 20.09 & 26.32 & LMCcascade w. filter & 3.27 & 1.0 & 900 \\
\hline 25.89 & 23.26 & 25.81 & 28.61 & SL w. filter & 0.981 & 1.0 & 270 \\
\hline 26.31 & 24.11 & 26.03 & 28.80 & LMCSL w. filter & 0.981 & 1.0 & 270 \\
\hline 26.66 & 28.80 & 27.81 & 23.37 & SL & 3.27 & 1.0 & 900 \\
\hline 26.74 & 24.61 & 27.02 & 28.60 & cascade w. filter & 0.981 & 1.0 & 270 \\
\hline 26.99 & 29.03 & 28.12 & 23.83 & cascade & 3.27 & 1.0 & 900 \\
\hline 28.70 & 25.69 & 28.94 & 31.48 & LMCcascade w. filter & 0.981 & 1.0 & 270 \\
\hline 29.36 & 32.72 & 29.21 & 26.16 & LMCSL & 3.27 & 1.0 & 900 \\
\hline 29.53 & 32.74 & 29.40 & 26.46 & LMCcascade & 3.27 & 1.0 & 900 \\
\hline 34.23 & 33.11 & 33.20 & 36.37 & LMCSL w. filter & 0.327 & 1.0 & 90 \\
\hline 34.24 & 32.31 & 34.36 & 36.05 & SL w. filter & 0.327 & 1.0 & 90 \\
\hline 34.62 & 32.81 & 34.17 & 36.88 & cascade w. filter & 0.327 & 1.0 & 90 \\
\hline 35.01 & 33.74 & 33.06 & 38.23 & LMCcascade w. filter & 0.327 & 1.0 & 90 \\
\hline 35.96 & 37.19 & 37.13 & 33.55 & SL & 0.981 & 1.0 & 270 \\
\hline 36.09 & 37.23 & 37.35 & 33.69 & cascade & 0.981 & 1.0 & 270 \\
\hline 36.25 & 37.60 & 37.41 & 33.74 & LMCSL & 0.981 & 1.0 & 270 \\
\hline 36.32 & 37.60 & 37.48 & 33.88 & LMCcascade & 0.981 & 1.0 & 270 \\
\hline 41.01 & 41.31 & 41.29 & 40.44 & SL & 0.327 & 1.0 & 90 \\
\hline 41.02 & 41.31 & 41.29 & 40.47 & cascade & 0.327 & 1.0 & 90 \\
\hline 41.04 & 41.35 & 41.29 & 40.47 & LMCcascade & 0.327 & 1.0 & 90 \\
\hline 41.05 & 41.35 & 41.29 & 40.51 & LMCSL & 0.327 & 1.0 & 90 \\
\hline
\end{tabular}

In Fig. 11 pure advection is considered. Table 6 shows the ranking for this test. Compared to the reference solution, the ASD scheme generates spurious waves around the top edges of the slotted cylinder, resulting in extreme values slightly different from the reference solution, namely 0.0572 and 5.022, compared to 0.2406 and 4.811. The semi-Lagrangian schemes are not possible to tell apart. The extreme values are exactly the same as those of the reference solution. All three schemes smooth the solution slightly, however, the shape of the cylinder is very well preserved. 
Table 2. Ranking results for the slotted cylinder with rural chemistry. Showing rank of the ranked error measures, rank of error measure $l_{1}$, $l_{2}$, and $l_{\infty}$ followed by method, maximum Courant number, grid resolution, and time step.

\begin{tabular}{|c|c|c|c|c|c|c|c|}
\hline $\operatorname{rank}($ all $)$ & $\operatorname{rank}\left(l_{1}\right)$ & $\operatorname{rank}\left(l_{2}\right)$ & $\operatorname{rank}\left(l_{\infty}\right)$ & Method & $\mathrm{CFL}_{\max }$ & $\Delta x$ & $\Delta t$ \\
\hline 4.56 & 2.83 & 3.16 & 7.69 & ASD w. filter & 0.327 & 0.5 & 45 \\
\hline 6.32 & 13.82 & 2.33 & 2.81 & ASD & 0.327 & 0.5 & 45 \\
\hline 9.69 & 10.31 & 13.25 & 5.49 & SL w. filter & 3.27 & 1.0 & 900 \\
\hline 10.07 & 11.47 & 13.25 & 5.49 & LMCSL w. filter & 3.27 & 1.0 & 900 \\
\hline 10.32 & 14.99 & 12.14 & 3.84 & ASD w. filter & 0.327 & 1.0 & 90 \\
\hline 12.40 & 26.91 & 7.81 & 2.48 & ASD & 0.327 & 1.0 & 90 \\
\hline 12.67 & 13.51 & 14.95 & 9.55 & SL w. filter & 0.981 & 1.0 & 270 \\
\hline 12.87 & 13.60 & 15.10 & 9.90 & LMCSL w. filter & 0.981 & 1.0 & 270 \\
\hline 14.07 & 2.01 & 4.85 & 35.35 & SL w. filter & 0.654 & 0.5 & 90 \\
\hline 14.07 & 15.27 & 16.69 & 10.27 & cascade w. filter & 0.981 & 1.0 & 270 \\
\hline 14.24 & 3.08 & 4.43 & 35.22 & LMCSL w. filter & 0.654 & 0.5 & 90 \\
\hline 14.97 & 17.00 & 18.17 & 9.75 & LMCcascade w. filter & 0.981 & 1.0 & 270 \\
\hline 15.02 & 4.41 & 5.43 & 35.24 & cascade w. filter & 0.654 & 0.5 & 90 \\
\hline 15.06 & 4.18 & 5.94 & 35.06 & LMCcascade w. filter & 0.654 & 0.5 & 90 \\
\hline 17.22 & 17.11 & 18.65 & 15.91 & cascade w. filter & 3.27 & 1.0 & 900 \\
\hline 19.19 & 6.82 & 9.63 & 41.13 & LMCSL w. filter & 0.327 & 0.5 & 45 \\
\hline 19.83 & 7.90 & 10.61 & 40.99 & cascade w. filter & 0.327 & 0.5 & 45 \\
\hline 19.93 & 8.41 & 10.00 & 41.37 & LMCcascade w. filter & 0.327 & 0.5 & 45 \\
\hline 20.12 & 21.95 & 21.14 & 17.27 & LMCcascade w. filter & 3.27 & 1.0 & 900 \\
\hline 20.15 & 8.81 & 9.90 & 41.76 & SL w. filter & 0.327 & 0.5 & 45 \\
\hline 25.50 & 26.29 & 26.92 & 23.30 & cascade & 0.654 & 0.5 & 90 \\
\hline 25.51 & 26.29 & 26.95 & 23.30 & LMCcascade & 0.654 & 0.5 & 90 \\
\hline 25.62 & 20.94 & 21.47 & 34.44 & SL w. filter & 0.327 & 1.0 & 90 \\
\hline 25.65 & 26.36 & 26.84 & 23.74 & SL & 0.654 & 0.5 & 90 \\
\hline 25.65 & 26.36 & 26.84 & 23.74 & LMCSL & 0.654 & 0.5 & 90 \\
\hline 25.75 & 20.95 & 21.96 & 34.33 & LMCSL w. filter & 0.327 & 1.0 & 90 \\
\hline 25.99 & 19.99 & 22.04 & 35.94 & cascade w. filter & 0.327 & 1.0 & 90 \\
\hline 26.10 & 20.16 & 22.32 & 35.81 & LMCcascade w. filter & 0.327 & 1.0 & 90 \\
\hline 26.82 & 33.21 & 33.53 & 13.71 & LMCSL & 3.27 & 1.0 & 900 \\
\hline 26.84 & 33.48 & 34.74 & 12.31 & LMCcascade & 3.27 & 1.0 & 900 \\
\hline 27.10 & 34.34 & 33.28 & 13.68 & SL & 3.27 & 1.0 & 900 \\
\hline 27.11 & 34.63 & 34.45 & 12.26 & cascade & 3.27 & 1.0 & 900 \\
\hline 30.09 & 30.15 & 30.85 & 29.28 & LMCcascade & 0.327 & 0.5 & 45 \\
\hline 30.11 & 30.20 & 30.85 & 29.28 & cascade & 0.327 & 0.5 & 45 \\
\hline 30.21 & 30.16 & 30.81 & 29.65 & SL & 0.327 & 0.5 & 45 \\
\hline 30.21 & 30.16 & 30.81 & 29.65 & LMCSL & 0.327 & 0.5 & 45 \\
\hline 32.43 & 38.34 & 37.77 & 21.18 & cascade & 0.981 & 1.0 & 270 \\
\hline 32.44 & 38.42 & 37.81 & 21.10 & LMCcascade & 0.981 & 1.0 & 270 \\
\hline 32.52 & 38.34 & 38.03 & 21.18 & SL & 0.981 & 1.0 & 270 \\
\hline 32.53 & 38.38 & 38.03 & 21.18 & LMCSL & 0.981 & 1.0 & 270 \\
\hline 35.65 & 41.85 & 41.53 & 23.56 & cascade & 0.327 & 1.0 & 90 \\
\hline 35.65 & 41.85 & 41.53 & 23.56 & LMCcascade & 0.327 & 1.0 & 90 \\
\hline 35.87 & 42.37 & 41.60 & 23.64 & SL & 0.327 & 1.0 & 90 \\
\hline 35.88 & 42.37 & 41.60 & 23.68 & LMCSL & 0.327 & 1.0 & 90 \\
\hline
\end{tabular}

\subsection{Error measures}

The following sections present the results obtained from ranking. Ranking is done by calculating error measures for each tracer and giving the points relative to their results.

In the tables below, the 44 tested methods have been ranked based on the error calculation described in
Eqs. (9-11). To achieve the ranks given in the tables, the three error measures were calculated for each of the $58 \mathrm{chem}$ ical species and every method. For every error measure and for every species, the best performing method was given the value 1 , the second best, the value 2 and so on up to the worst performing method, which was given the value 44 . For every method and for every error measure the mean value was 
calculated and given in the tables. Furthermore, a mean rank, based on the different ranks obtained for each of the different methods was found, rank(all). The best performing method is the method with the lowest value of $\operatorname{rank}($ all).

\subsubsection{Rotating cone with rural chemistry}

In Table 1 the error measures for the rotating cone with rural chemistry are ranked. It is seen in the table that the ASD scheme performs best, also, the finer the resolution the better the performance. The non-filtered solutions of both ASD and SL perform better on an overall consideration. When considering the individual errors, it is seen that the $l_{1}$ error is smaller for the filtered solution than for the non-filtered ASD.

The semi-Lagrangian schemes perform better with filter and a fine spacial resolution with big time step. Among the semi-Lagrangian schemes, the LMCSL scheme with filter performs best for the two finest resolutions in space and time. The highest Courant number, 3.27, and resolution 3_1, $\Delta x=0.5$ and $\Delta t=90$. perform equally well. The worst results for the semi-Lagrangian schemes are obtained using the highest courant number less than 1, resolution 3_1, or the traditional DEHM resolution, $\Delta x=1.0$ and $\Delta t=90$. For the finest resolution, the four unfiltered semi-Lagrangian schemes perform equally well, the same is the case for the fine resolution with a coarser time step and for the resolution traditionally used in DEHM.

\subsubsection{Slotted cylinder with rural chemistry}

The results obtained for the slotted cylinder with rural chemistry, Table 2, are quite different from the previous. All nonfiltered semi-Lagrangian solutions are in the bottom of the table, sorted only by resolution, resolution $1 \_05$ performs best, followed by the two filtered cascade schemes using resolution 1_1. The second best performing resolution is 10_1, third is resolution 05_05, fourth is 3_1 and last is resolution $1 \_1$, the resolution used in DEHM.

Considering the ASD scheme, it can be seen that the filtered solution now performs better than the unfiltered solution. This is due to the very sharp gradients in the slotted cylinder and the wiggles the scheme creates when performing the advection.

Of the semi-Lagrangian schemes the filtered classical semi-Lagrangian solution and the filtered LMCSL scheme, both with resolution 10_1, perform best and, on an overall basis, better than the ASD schemes with resolution 1_1.

The second group of filtered semi-Lagrangian schemes are the schemes using resolutions 3_1 and 1_05 followed by the two filtered cascade schemes using the coarsest resolution and the filtered resolution 05_05. The results achieved using the resolution used in DEHM gives the lowest score, even worse than the non-filtered resolution 1_05 for most cases.
Almost exactly as is the case for the distribution of the nonfiltered schemes.

When considering the $l_{1}$ error it is seen that the best performing scheme is the classical semi-Lagrangian scheme with filter, resolution 1_05. The second best considering this error is the overall best ranked scheme for this test, namely the ASD with filter resolution 05_05, the third best scheme is the filtered LMCSL scheme using resolution 1_05.

The best solution with regard to the $l_{2}$ error is the nonfiltered ASD scheme with fine resolution. As above, the second best performing scheme is the filtered ASD the with same resolution. The third best is the filtered LMCSL with resolution 1_05.

The overall worst performing of the ASD schemes, the non-filtered ASD with resolution 1_1, performs best with regard to the $l_{\infty}$ error. The fine resolution non-filtered ASD scheme scores the second best rank, and the third best is the ASD with filter using the traditional resolution.

\subsubsection{Rotating cone with urban chemistry}

Table 3 shows the ranking results from testing the rotating cone with urban chemistry. For this test, the high resolution filtered ASD scheme performs best followed by the semi-Lagrangian schemes using resolution 1_05 and the filtered semi-Lagrangian schemes with resolution 10_1. After these, the non-filtered high resolution ASD and the semiLagrangian schemes with resolution 05_05 follow, along with the remaining semi-Lagrangian schemes using resolution 10_1.

Considering the individual errors, the result is different. According to the $l_{1}$ error the best ranked scheme is the filtered LMC cascade scheme using resolution 1_05, second is the semi-Lagrangian interpolation with filter and third is the LMCSL scheme with filter, both using the same resolution as the first.

Also for the $l_{2}$ error, the filtered LMC cascade scheme with resolution 1_05 gives the best rank, the second best according to this rank is the second best according to the $l_{1}$ error, as well, namely the filtered classical semi-Lagrangian interpolation with resolution 1_05. The filtered ASD with resolution $05 \_05$, third according to the $l_{2}$ error, is the best scheme according to the overall rank.

The only error for which the overall best scheme actually scores best is the ranking of the $l_{\infty}$ error. After the filtered high resolution ASD, LMC cascade scores second and pure cascade comes third, both of the latter with resolution 1_05.

As with the previous rankings, the non-filtered semiLagrangian schemes are in the bottom of the table, however, for this test the two filtered classical semi-Lagrangian schemes using resolution $1 \_1$ are the worst performing schemes.

The two ASD schemes using the traditional resolution, $1 \_1$, are also placed rather low in the table, both according 
Table 3. Raking results for the rotating cone with urban chemistry. Showing rank of the ranked error measures, rank of error measure $l_{1}, l_{2}$, and $l_{\infty}$ followed by method, maximum Courant number, grid resolution, and time step.

\begin{tabular}{|c|c|c|c|c|c|c|c|}
\hline $\operatorname{rank}($ all $)$ & $\operatorname{rank}\left(l_{1}\right)$ & $\operatorname{rank}\left(l_{2}\right)$ & $\operatorname{rank}\left(l_{\infty}\right)$ & Method & $\mathrm{CFL}_{\max }$ & $\Delta x$ & $\Delta t$ \\
\hline 7.77 & 9.16 & 8.59 & 5.55 & ASD w. filter & 0.327 & 0.5 & 45 \\
\hline 9.04 & 7.36 & 8.15 & 11.61 & LMCcascade w. filter & 0.654 & 0.5 & 90 \\
\hline 9.31 & 7.39 & 8.49 & 12.05 & SL w. filter & 0.654 & 0.5 & 90 \\
\hline 9.39 & 7.71 & 8.77 & 11.69 & LMCSL w. filter & 0.654 & 0.5 & 90 \\
\hline 9.47 & 8.00 & 8.63 & 11.77 & cascade w. filter & 0.654 & 0.5 & 90 \\
\hline 11.84 & 13.27 & 11.86 & 10.38 & LMCcascade & 0.654 & 0.5 & 90 \\
\hline 11.84 & 13.27 & 11.86 & 10.40 & cascade & 0.654 & 0.5 & 90 \\
\hline 11.92 & 13.23 & 11.86 & 10.66 & SL & 0.654 & 0.5 & 90 \\
\hline 11.98 & 9.67 & 11.07 & 15.19 & SL w. filter & 3.27 & 1.0 & 900 \\
\hline 12.02 & 13.27 & 11.90 & 10.90 & LMCSL & 0.654 & 0.5 & 90 \\
\hline 12.68 & 11.37 & 10.98 & 15.69 & LMCSL w. filter & 3.27 & 1.0 & 900 \\
\hline 12.84 & 14.72 & 12.93 & 10.86 & ASD & 0.327 & 0.5 & 45 \\
\hline 16.09 & 14.02 & 16.56 & 17.69 & cascade w. filter & 3.27 & 1.0 & 900 \\
\hline 17.05 & 18.18 & 16.95 & 16.02 & cascade & 0.327 & 0.5 & 45 \\
\hline 17.12 & 18.25 & 17.03 & 16.09 & LMCcascade & 0.327 & 0.5 & 45 \\
\hline 17.13 & 18.18 & 16.99 & 16.22 & LMCSL & 0.327 & 0.5 & 45 \\
\hline 17.15 & 18.21 & 16.99 & 16.26 & SL & 0.327 & 0.5 & 45 \\
\hline 17.30 & 14.87 & 14.86 & 22.18 & LMCcascade w. filter & 3.27 & 1.0 & 900 \\
\hline 17.48 & 14.62 & 16.97 & 20.86 & LMCSL w. filter & 0.327 & 0.5 & 45 \\
\hline 17.70 & 14.72 & 17.10 & 21.29 & cascade w. filter & 0.327 & 0.5 & 45 \\
\hline 17.86 & 14.81 & 17.18 & 21.61 & LMCcascade w. filter & 0.327 & 0.5 & 45 \\
\hline 18.05 & 14.89 & 17.30 & 21.97 & SL w. filter & 0.327 & 0.5 & 45 \\
\hline 20.94 & 22.62 & 22.92 & 17.27 & ASD w. filter & 0.327 & 1.0 & 90 \\
\hline 21.41 & 22.25 & 22.17 & 19.81 & SL & 3.27 & 1.0 & 900 \\
\hline 21.84 & 22.49 & 23.01 & 20.01 & cascade & 3.27 & 1.0 & 900 \\
\hline 22.96 & 24.47 & 23.30 & 21.11 & LMCSL & 3.27 & 1.0 & 900 \\
\hline 23.33 & 24.83 & 23.85 & 21.30 & LMCcascade & 3.27 & 1.0 & 900 \\
\hline 27.23 & 31.90 & 27.86 & 21.93 & ASD & 0.327 & 1.0 & 90 \\
\hline 27.23 & 27.61 & 27.20 & 26.87 & SL w. filter & 0.981 & 1.0 & 270 \\
\hline 27.54 & 27.91 & 27.63 & 27.08 & LMCSL w. filter & 0.981 & 1.0 & 270 \\
\hline 28.35 & 29.07 & 28.85 & 27.13 & cascade w. filter & 0.981 & 1.0 & 270 \\
\hline 28.76 & 28.34 & 28.88 & 29.06 & LMCcascade w. filter & 0.981 & 1.0 & 270 \\
\hline 32.63 & 33.22 & 33.44 & 31.22 & LMCSL & 0.981 & 1.0 & 270 \\
\hline 32.64 & 33.19 & 33.48 & 31.24 & SL & 0.981 & 1.0 & 270 \\
\hline 32.81 & 33.73 & 33.48 & 31.21 & cascade & 0.981 & 1.0 & 270 \\
\hline 32.86 & 33.69 & 33.60 & 31.29 & LMCcascade & 0.981 & 1.0 & 270 \\
\hline 37.56 & 36.31 & 37.54 & 38.85 & cascade w. filter & 0.327 & 1.0 & 90 \\
\hline 37.57 & 36.44 & 37.45 & 38.83 & LMCcascade w. filter & 0.327 & 1.0 & 90 \\
\hline 38.32 & 39.32 & 38.41 & 37.22 & cascade & 0.327 & 1.0 & 90 \\
\hline 38.32 & 39.32 & 38.41 & 37.24 & LMCcascade & 0.327 & 1.0 & 90 \\
\hline 38.48 & 39.36 & 38.44 & 37.64 & SL & 0.327 & 1.0 & 90 \\
\hline 38.49 & 39.36 & 38.44 & 37.68 & LMCSL & 0.327 & 1.0 & 90 \\
\hline 38.84 & 37.88 & 39.33 & 39.31 & SL w. filter & 0.327 & 1.0 & 90 \\
\hline 38.86 & 37.53 & 39.28 & 39.77 & LMCSL w. filter & 0.327 & 1.0 & 90 \\
\hline
\end{tabular}

to the overall rank and the individual ranking of the error measures.

\subsubsection{Slotted cylinder with urban chemistry}

Table 4 shows the results of the most severe test with the most difficult chemistry, namely the slotted cylinder with urban chemistry. The semi-Lagrangian schemes with the coarsest resolution give the best performance, with the filtered schemes first followed by the unfiltered, and the filtered resolution 3_1 follow. The next "group" is the nonfiltered semi-Lagrangian schemes using resolution 1_05 followed by the same schemes with resolution 05_05. The filtered semi-Lagrangian schemes using resolution 1_05 and 
Table 4. Ranking results for the slotted cylinder with urban chemistry. Showing rank of the ranked error measures, rank of error measure $l_{1}$, $l_{2}$, and $l_{\infty}$ followed by method, maximum Courant number, grid resolution, and time step.

\begin{tabular}{|c|c|c|c|c|c|c|c|}
\hline $\operatorname{rank}($ all $)$ & $\operatorname{rank}\left(l_{1}\right)$ & $\operatorname{rank}\left(l_{2}\right)$ & $\operatorname{rank}\left(l_{\infty}\right)$ & Method & $\mathrm{CFL}_{\max }$ & $\Delta x$ & $\Delta t$ \\
\hline 3.64 & 2.79 & 3.24 & 4.90 & SL w. filter & 3.27 & 1.0 & 900 \\
\hline 3.97 & 3.42 & 3.48 & 5.01 & LMCSL w. filter & 3.27 & 1.0 & 900 \\
\hline 7.23 & 4.31 & 5.83 & 11.54 & cascade w. filter & 3.27 & 1.0 & 900 \\
\hline 7.56 & 5.39 & 6.49 & 10.81 & LMCcascade w. filter & 3.27 & 1.0 & 900 \\
\hline 14.85 & 19.91 & 16.01 & 8.63 & LMCSL & 3.27 & 1.0 & 900 \\
\hline 14.91 & 20.77 & 15.55 & 8.42 & SL & 3.27 & 1.0 & 900 \\
\hline 15.26 & 20.90 & 17.54 & 7.34 & LMCcascade & 3.27 & 1.0 & 900 \\
\hline 15.41 & 13.88 & 15.63 & 16.72 & LMCcascade w. filter & 0.981 & 1.0 & 270 \\
\hline 15.42 & 21.83 & 17.22 & 7.19 & cascade & 3.27 & 1.0 & 900 \\
\hline 15.90 & 14.18 & 16.24 & 17.29 & cascade w. filter & 0.981 & 1.0 & 270 \\
\hline 16.07 & 14.40 & 16.23 & 17.58 & SL w. filter & 0.981 & 1.0 & 270 \\
\hline 16.19 & 14.68 & 16.69 & 17.21 & LMCSL w. filter & 0.981 & 1.0 & 270 \\
\hline 17.33 & 17.78 & 14.49 & 19.72 & SL & 0.654 & 0.5 & 90 \\
\hline 17.33 & 17.78 & 14.49 & 19.72 & LMCSL & 0.654 & 0.5 & 90 \\
\hline 17.59 & 18.14 & 15.11 & 19.52 & LMCcascade & 0.654 & 0.5 & 90 \\
\hline 17.60 & 18.14 & 15.13 & 19.52 & cascade & 0.654 & 0.5 & 90 \\
\hline 20.52 & 21.82 & 18.21 & 21.52 & SL & 0.327 & 0.5 & 45 \\
\hline 20.53 & 21.82 & 18.29 & 21.48 & LMCSL & 0.327 & 0.5 & 45 \\
\hline 20.61 & 21.86 & 18.69 & 21.28 & LMCcascade & 0.327 & 0.5 & 45 \\
\hline 20.62 & 21.90 & 18.66 & 21.31 & cascade & 0.327 & 0.5 & 45 \\
\hline 22.92 & 13.66 & 20.79 & 34.31 & LMCSL w. filter & 0.654 & 0.5 & 90 \\
\hline 23.29 & 14.43 & 21.55 & 33.90 & SL w. filter & 0.654 & 0.5 & 90 \\
\hline 23.52 & 14.73 & 21.55 & 34.30 & LMCcascade w. filter & 0.654 & 0.5 & 90 \\
\hline 24.32 & 15.51 & 22.31 & 35.15 & cascade w. filter & 0.654 & 0.5 & 90 \\
\hline 24.69 & 31.39 & 27.03 & 15.64 & LMCSL & 0.981 & 1.0 & 270 \\
\hline 24.73 & 31.41 & 27.10 & 15.68 & SL & 0.981 & 1.0 & 270 \\
\hline 25.47 & 32.18 & 27.89 & 16.35 & cascade & 0.981 & 1.0 & 270 \\
\hline 25.49 & 32.16 & 27.91 & 16.41 & LMCcascade & 0.981 & 1.0 & 270 \\
\hline 27.47 & 25.26 & 26.40 & 30.75 & ASD w. filter & 0.327 & 0.5 & 45 \\
\hline 28.94 & 37.16 & 31.67 & 17.99 & SL & 0.327 & 1.0 & 90 \\
\hline 28.94 & 37.18 & 31.67 & 17.99 & LMCSL & 0.327 & 1.0 & 90 \\
\hline 29.00 & 37.03 & 31.73 & 18.25 & LMCcascade & 0.327 & 1.0 & 90 \\
\hline 29.03 & 37.10 & 31.75 & 18.25 & cascade & 0.327 & 1.0 & 90 \\
\hline 30.02 & 33.52 & 31.56 & 24.98 & ASD w. filter & 0.327 & 1.0 & 90 \\
\hline 30.22 & 26.88 & 30.32 & 33.45 & LMCcascade w. filter & 0.327 & 1.0 & 90 \\
\hline 30.27 & 26.89 & 31.19 & 32.72 & SL w. filter & 0.327 & 1.0 & 90 \\
\hline 30.84 & 27.71 & 31.30 & 33.51 & cascade w. filter & 0.327 & 1.0 & 90 \\
\hline 30.94 & 27.61 & 31.70 & 33.50 & LMCSL w. filter & 0.327 & 1.0 & 90 \\
\hline 30.95 & 22.50 & 30.41 & 39.94 & cascade w. filter & 0.327 & 0.5 & 45 \\
\hline 30.96 & 22.51 & 30.63 & 39.74 & SL w. filter & 0.327 & 0.5 & 45 \\
\hline 31.10 & 22.71 & 30.64 & 39.94 & LMCcascade w. filter & 0.327 & 0.5 & 45 \\
\hline 31.77 & 23.29 & 31.38 & 40.63 & LMCSL w. filter & 0.327 & 0.5 & 45 \\
\hline 37.91 & 39.81 & 38.46 & 35.44 & ASD & 0.327 & 0.5 & 45 \\
\hline 38.67 & 41.70 & 39.85 & 34.46 & ASD & 0.327 & 1.0 & 90 \\
\hline
\end{tabular}

non-filtered resolution 3_1 precede the filtered ASD scheme with the finest resolution. The schemes using resolution 1_1 including the filtered ASD are next, followed only by the filtered semi-Lagrangian schemes using resolution 05_05 and the two non-filtered ASD schemes.

When considering the individual error ranks, the three best methods are exactly the same for the overall rank and for the rank of the $l_{1}$ and $l_{2}$ errors. For the rank of the $l_{\infty}$ error, the two best schemes are again the same, but the third best according to this error measure is a bit further down the list; but with the same resolution as the others. The two best performing schemes, regardless of which rank is considered, are the filtered semi-Lagrangian scheme and the filtered LMCSL scheme, both with resolution 10_1. The third best scheme 
Table 5. Ranking results for the rotating cone with pure advection. Showing rank of the ranked error measures, rank of error measure $l_{1}, l_{2}$, and $l_{\infty}$ followed by method, maximum Courant number, grid resolution, and time step.

\begin{tabular}{|c|c|c|c|c|c|c|c|}
\hline $\operatorname{rank}($ all $)$ & $\operatorname{rank}\left(l_{1}\right)$ & $\operatorname{rank}\left(l_{2}\right)$ & $\operatorname{rank}\left(l_{\infty}\right)$ & Method & $\mathrm{CFL}_{\max }$ & $\Delta x$ & $\Delta t$ \\
\hline 1.46 & 1.00 & 2.00 & 1.38 & ASD w. filter & 0.327 & 0.5 & 45 \\
\hline 1.54 & 2.00 & 1.00 & 1.63 & ASD & 0.327 & 0.5 & 45 \\
\hline 3.33 & 4.00 & 3.00 & 3.00 & ASD & 0.327 & 1.0 & 90 \\
\hline 3.67 & 3.00 & 4.00 & 4.00 & ASD w. filter & 0.327 & 1.0 & 90 \\
\hline 5.89 & 6.04 & 5.83 & 5.79 & LMCSL w. filter & 0.654 & 0.5 & 90 \\
\hline 5.94 & 6.38 & 5.67 & 5.79 & SL w. filter & 0.654 & 0.5 & 90 \\
\hline 6.79 & 6.88 & 6.96 & 6.54 & cascade w. filter & 0.654 & 0.5 & 90 \\
\hline 7.38 & 6.71 & 7.54 & 7.88 & LMCcascade w. filter & 0.654 & 0.5 & 90 \\
\hline 10.96 & 9.96 & 11.29 & 11.63 & SL w. filter & 0.327 & 0.5 & 45 \\
\hline 14.49 & 12.75 & 9.63 & 21.08 & SL w. filter & 3.27 & 1.0 & 900 \\
\hline 14.49 & 11.25 & 13.50 & 18.71 & cascade w. filter & 0.327 & 0.5 & 45 \\
\hline 14.83 & 10.92 & 14.38 & 19.21 & LMCcascade w. filter & 0.327 & 0.5 & 45 \\
\hline 14.88 & 12.88 & 16.54 & 15.21 & LMCSL w. filter & 0.327 & 0.5 & 45 \\
\hline 15.39 & 13.75 & 10.33 & 22.08 & LMCSL w. filter & 3.27 & 1.0 & 900 \\
\hline 15.93 & 18.25 & 17.29 & 12.25 & cascade & 0.654 & 0.5 & 90 \\
\hline 15.93 & 18.25 & 17.29 & 12.25 & LMCcascade & 0.654 & 0.5 & 90 \\
\hline 15.93 & 18.25 & 17.29 & 12.25 & SL & 0.654 & 0.5 & 90 \\
\hline 15.93 & 18.25 & 17.29 & 12.25 & LMCSL & 0.654 & 0.5 & 90 \\
\hline 16.83 & 15.67 & 19.17 & 15.67 & cascade w. filter & 3.27 & 1.0 & 900 \\
\hline 19.36 & 15.67 & 14.67 & 27.75 & LMCcascade w. filter & 3.27 & 1.0 & 900 \\
\hline 22.25 & 26.29 & 22.25 & 18.21 & cascade & 0.327 & 0.5 & 45 \\
\hline 22.25 & 26.29 & 22.25 & 18.21 & LMCcascade & 0.327 & 0.5 & 45 \\
\hline 22.25 & 26.29 & 22.25 & 18.21 & SL & 0.327 & 0.5 & 45 \\
\hline 22.25 & 26.29 & 22.25 & 18.21 & LMCSL & 0.327 & 0.5 & 45 \\
\hline 25.57 & 21.63 & 25.33 & 29.75 & SL w. filter & 0.981 & 1.0 & 270 \\
\hline 25.97 & 22.46 & 25.71 & 29.75 & LMCSL w. filter & 0.981 & 1.0 & 270 \\
\hline 26.21 & 22.38 & 26.50 & 29.75 & cascade w. filter & 0.981 & 1.0 & 270 \\
\hline 27.25 & 22.54 & 27.46 & 31.75 & LMCcascade w. filter & 0.981 & 1.0 & 270 \\
\hline 27.64 & 29.50 & 29.71 & 23.71 & SL & 3.27 & 1.0 & 900 \\
\hline 27.79 & 29.50 & 29.71 & 24.17 & cascade & 3.27 & 1.0 & 900 \\
\hline 29.86 & 33.83 & 29.88 & 25.88 & LMCSL & 3.27 & 1.0 & 900 \\
\hline 30.65 & 33.83 & 31.71 & 26.42 & LMCcascade & 3.27 & 1.0 & 900 \\
\hline 34.32 & 32.08 & 33.50 & 37.38 & LMCSL w. filter & 0.327 & 1.0 & 90 \\
\hline 35.38 & 34.04 & 34.54 & 37.54 & SL w. filter & 0.327 & 1.0 & 90 \\
\hline 35.74 & 33.29 & 34.08 & 39.83 & LMCcascade w. filter & 0.327 & 1.0 & 90 \\
\hline 35.79 & 33.92 & 34.88 & 38.58 & cascade w. filter & 0.327 & 1.0 & 90 \\
\hline 36.83 & 38.29 & 38.21 & 34.00 & SL & 0.981 & 1.0 & 270 \\
\hline 37.00 & 38.46 & 38.38 & 34.17 & cascade & 0.981 & 1.0 & 270 \\
\hline 37.06 & 38.63 & 38.38 & 34.17 & LMCSL & 0.981 & 1.0 & 270 \\
\hline 37.22 & 38.63 & 38.38 & 34.67 & LMCcascade & 0.981 & 1.0 & 270 \\
\hline 42.44 & 42.50 & 42.50 & 42.33 & cascade & 0.327 & 1.0 & 90 \\
\hline 42.44 & 42.50 & 42.50 & 42.33 & LMCcascade & 0.327 & 1.0 & 90 \\
\hline 42.44 & 42.50 & 42.50 & 42.33 & SL & 0.327 & 1.0 & 90 \\
\hline 42.44 & 42.50 & 42.50 & 42.33 & LMCSL & 0.327 & 1.0 & 90 \\
\hline
\end{tabular}

according to the overall error rank and the $l_{1}$ and $l_{2}$ error ranks is the filtered cascade interpolation. Pure cascade interpolation scores best with regard to the rank of the $l_{\infty}$ error.

\subsubsection{Pure advection of the rotating cone}

The ranks of the schemes when tested on pure advection of the rotating cone are shown in Table 5. It is seen that for this test the ASD scheme is the ultimate scheme. The four ASD schemes give the best scores for both the overall and the individual ranks. 
Table 6. Ranking results for the slotted cylinder with pure advection. Showing rank of the ranked error measures, rank of error measure $l_{1}$, $l_{2}$, and $l_{\infty}$ followed by method, maximum Courant number, grid resolution, and time step.

\begin{tabular}{|c|c|c|c|c|c|c|c|}
\hline $\operatorname{rank}($ all $)$ & $\operatorname{rank}\left(l_{1}\right)$ & $\operatorname{rank}\left(l_{2}\right)$ & $\operatorname{rank}\left(l_{\infty}\right)$ & Method & $\mathrm{CFL}_{\max }$ & $\Delta x$ & $\Delta t$ \\
\hline 4.33 & 10.00 & 1.00 & 2.00 & ASD & 0.327 & 0.5 & 45 \\
\hline 7.25 & 8.75 & 3.00 & 10.00 & ASD w. filter & 0.327 & 0.5 & 45 \\
\hline 9.00 & 24.00 & 2.00 & 1.00 & ASD & 0.327 & 1.0 & 90 \\
\hline 9.56 & 11.00 & 13.50 & 4.17 & SL w. filter & 3.27 & 1.0 & 900 \\
\hline 10.11 & 12.00 & 13.50 & 4.83 & LMCSL w. filter & 3.27 & 1.0 & 900 \\
\hline 10.67 & 17.00 & 12.00 & 3.00 & ASD w. filter & 0.327 & 1.0 & 90 \\
\hline 12.25 & 13.29 & 15.21 & 8.25 & LMCSL w. filter & 0.981 & 1.0 & 270 \\
\hline 12.75 & 13.71 & 15.79 & 8.75 & SL w. filter & 0.981 & 1.0 & 270 \\
\hline 13.00 & 15.00 & 17.00 & 7.00 & cascade w. filter & 0.981 & 1.0 & 270 \\
\hline 13.33 & 16.00 & 18.00 & 6.00 & LMCcascade w. filter & 0.981 & 1.0 & 270 \\
\hline 14.46 & 1.38 & 4.58 & 37.42 & LMCSL w. filter & 0.654 & 0.5 & 90 \\
\hline 14.93 & 1.63 & 4.42 & 38.75 & SL w. filter & 0.654 & 0.5 & 90 \\
\hline 15.46 & 3.33 & 6.50 & 36.54 & LMCcascade w. filter & 0.654 & 0.5 & 90 \\
\hline 15.83 & 3.67 & 6.50 & 37.33 & cascade w. filter & 0.654 & 0.5 & 90 \\
\hline 17.74 & 19.21 & 19.00 & 15.00 & cascade w. filter & 3.27 & 1.0 & 900 \\
\hline 19.14 & 5.92 & 9.00 & 42.50 & SL w. filter & 0.327 & 0.5 & 45 \\
\hline 19.25 & 6.42 & 8.83 & 42.50 & LMCSL w. filter & 0.327 & 0.5 & 45 \\
\hline 19.67 & 23.00 & 20.00 & 16.00 & LMCcascade w. filter & 3.27 & 1.0 & 900 \\
\hline 19.67 & 6.54 & 9.96 & 42.50 & LMCcascade w. filter & 0.327 & 0.5 & 45 \\
\hline 20.03 & 7.38 & 10.21 & 42.50 & cascade w. filter & 0.327 & 0.5 & 45 \\
\hline 24.69 & 19.17 & 21.83 & 33.08 & SL w. filter & 0.327 & 1.0 & 90 \\
\hline 25.69 & 20.67 & 22.50 & 33.92 & LMCSL w. filter & 0.327 & 1.0 & 90 \\
\hline 26.17 & 26.50 & 26.50 & 25.50 & cascade & 0.654 & 0.5 & 90 \\
\hline 26.17 & 26.50 & 26.50 & 25.50 & LMCcascade & 0.654 & 0.5 & 90 \\
\hline 26.22 & 20.38 & 22.38 & 35.92 & LMCcascade w. filter & 0.327 & 1.0 & 90 \\
\hline 26.47 & 33.00 & 33.42 & 13.00 & $\mathrm{SL}$ & 3.27 & 1.0 & 900 \\
\hline 26.71 & 34.00 & 35.13 & 11.00 & cascade & 3.27 & 1.0 & 900 \\
\hline 26.83 & 26.50 & 26.50 & 27.50 & SL & 0.654 & 0.5 & 90 \\
\hline 26.83 & 26.50 & 26.50 & 27.50 & LMCSL & 0.654 & 0.5 & 90 \\
\hline 27.54 & 35.00 & 33.63 & 14.00 & LMCSL & 3.27 & 1.0 & 900 \\
\hline 27.64 & 20.58 & 23.29 & 39.04 & cascade w. filter & 0.327 & 1.0 & 90 \\
\hline 27.94 & 36.00 & 35.83 & 12.00 & LMCcascade & 3.27 & 1.0 & 900 \\
\hline 30.25 & 30.50 & 30.33 & 29.92 & cascade & 0.327 & 0.5 & 45 \\
\hline 30.25 & 30.50 & 30.33 & 29.92 & LMCcascade & 0.327 & 0.5 & 45 \\
\hline 30.75 & 30.50 & 30.67 & 31.08 & SL & 0.327 & 0.5 & 45 \\
\hline 30.75 & 30.50 & 30.67 & 31.08 & LMCSL & 0.327 & 0.5 & 45 \\
\hline 31.38 & 38.38 & 38.25 & 17.50 & LMCcascade & 0.981 & 1.0 & 270 \\
\hline 31.43 & 38.54 & 38.25 & 17.50 & cascade & 0.981 & 1.0 & 270 \\
\hline 32.26 & 38.54 & 38.75 & 19.50 & SL & 0.981 & 1.0 & 270 \\
\hline 32.26 & 38.54 & 38.75 & 19.50 & LMCSL & 0.981 & 1.0 & 270 \\
\hline 35.31 & 41.75 & 42.42 & 21.75 & cascade & 0.327 & 1.0 & 90 \\
\hline 35.31 & 41.75 & 42.42 & 21.75 & LMCcascade & 0.327 & 1.0 & 90 \\
\hline 36.36 & 43.25 & 42.58 & 23.25 & SL & 0.327 & 1.0 & 90 \\
\hline 36.36 & 43.25 & 42.58 & 23.25 & LMCSL & 0.327 & 1.0 & 90 \\
\hline
\end{tabular}

The following schemes are, grouped by resolution, the filtered semi-Lagrangian schemes with resolution 1_05, followed by the same schemes with resolution 05_05, and the filtered classical semi-Lagrangian scheme with the coarsest resolution, i.e. 10_1. Then follow the filtered LMCSL scheme and the unfiltered solutions of resolution 1_05, the filtered coarse resolution cascade schemes, the non-filtered schemes with resolution $05 \_05$, the filtered resolution 3_1, and resolution 10_1. Last are the filtered schemes using resolution 1_1, the non-filtered resolution 3_1, and the remaining resolution 1_1.

The individually ranked errors place the four ASD schemes as the top schemes as well as the overall rank. For the rank of the $l_{1}$ error, the four best performing schemes 
Table 7. Ranking results for the sum of rank(all) from all six ranking tables. Showing the sum of the total rank, rank(all(all)), method, maximum courant number, grid size, and time step.

\begin{tabular}{|c|c|c|c|c|}
\hline $\operatorname{rank}(\operatorname{all}($ all $))$ & Method & $\mathrm{CFL}_{\max }$ & $\Delta x$ & $\Delta t$ \\
\hline 50.18 & ASD w. filter & 0.327 & 0.5 & 45 \\
\hline 64.05 & SL w. filter & 3.27 & 1.0 & 900 \\
\hline 64.40 & ASD & 0.327 & 0.5 & 45 \\
\hline 67.94 & LMCSL w. filter & 3.27 & 1.0 & 900 \\
\hline 74.55 & LMCSL w. filter & 0.654 & 0.5 & 90 \\
\hline 75.31 & SL w. filter & 0.654 & 0.5 & 90 \\
\hline 79.47 & ASD w. filter & 0.327 & 1.0 & 90 \\
\hline 79.58 & LMCcascade w. filter & 0.654 & 0.5 & 90 \\
\hline 80.16 & cascade w. filter & 0.654 & 0.5 & 90 \\
\hline 93.21 & cascade w. filter & 3.27 & 1.0 & 900 \\
\hline 94.16 & ASD & 0.327 & 1.0 & 90 \\
\hline 105.95 & LMCcascade w. filter & 3.27 & 1.0 & 900 \\
\hline 112.52 & cascade & 0.654 & 0.5 & 90 \\
\hline 112.56 & LMCcascade & 0.654 & 0.5 & 90 \\
\hline 113.13 & SL & 0.654 & 0.5 & 90 \\
\hline 113.24 & LMCSL & 0.654 & 0.5 & 90 \\
\hline 115.69 & SL w. filter & 0.327 & 0.5 & 45 \\
\hline 116.36 & LMCSL w. filter & 0.327 & 0.5 & 45 \\
\hline 118.83 & LMCcascade w. filter & 0.327 & 0.5 & 45 \\
\hline 119.00 & cascade w. filter & 0.327 & 0.5 & 45 \\
\hline 120.18 & SL w. filter & 0.981 & 1.0 & 270 \\
\hline 121.13 & LMCSL w. filter & 0.981 & 1.0 & 270 \\
\hline 125.31 & cascade w. filter & 0.981 & 1.0 & 270 \\
\hline 127.38 & LMCcasc w filter & 0.981 & 1.0 & 270 \\
\hline 141.02 & cascade & 0.327 & 0.5 & 45 \\
\hline 141.06 & LMCcascade & 0.327 & 0.5 & 45 \\
\hline 141.61 & LMCSL & 0.327 & 0.5 & 45 \\
\hline 141.62 & SL & 0.327 & 0.5 & 45 \\
\hline 144.19 & SL & 3.27 & 1.0 & 900 \\
\hline 145.86 & cascade & 3.27 & 1.0 & 900 \\
\hline 151.39 & LMCSL & 3.27 & 1.0 & 900 \\
\hline 153.55 & LMCcascade & 3.27 & 1.0 & 900 \\
\hline 189.04 & SL w. filter & 0.327 & 1.0 & 90 \\
\hline 189.79 & LMCSL w. filter & 0.327 & 1.0 & 90 \\
\hline 190.86 & LMCcascade w. filter & 0.327 & 1.0 & 90 \\
\hline 192.44 & cascade w. filter & 0.327 & 1.0 & 90 \\
\hline 194.42 & LMCSL & 0.981 & 1.0 & 270 \\
\hline 194.94 & SL & 0.981 & 1.0 & 270 \\
\hline 195.23 & cascade & 0.981 & 1.0 & 270 \\
\hline 195.71 & LMCcasc & 0.981 & 1.0 & 270 \\
\hline 221.76 & LMCcasc & 0.327 & 1.0 & 90 \\
\hline 221.77 & cascade & 0.327 & 1.0 & 90 \\
\hline 223.10 & SL & 0.327 & 1.0 & 90 \\
\hline 223.16 & LMCSL & 0.327 & 1.0 & 90 \\
\hline
\end{tabular}

are, in order of best to worst, ASD with filter using the finest resolution, the non-filtered solution of the ASD scheme using the finest resolution, the filtered coarse resolution ASD and the non-filtered.

Considering the rank of the $l_{2}$ error, the first two schemes switch position, the non-filtered fine resolution now is the best followed by the filtered fine resolution and the same order for the coarse resolution.
The last error measure, the $l_{\infty}$, ranks the schemes in the same order as the overall rank: first the filtered fine resolution, then the non-filtered, third the non-filtered coarse resolution and finally the filtered coarse resolution ASD. 


\subsubsection{Pure advection of the slotted cylinder}

The last test, Table 6 shows pure advection of the slotted cylinder. Again the two fine resolution ASD schemes give the best overall rank, however, not as unanimously as above.

The ASD schemes using the fine resolution and the nonfiltered coarse resolution are followed by the coarse resolution filtered SL and LMCSL schemes and the remaining ASD. Then, the filtered 3_1 and 1_05 resolution semiLagrangian schemes follow, these are followed by the filtered coarse resolution cascade and LMC cascade scheme and the filtered resolution 05_05. The next group consists of the filtered resolution 1_1, non-filtered resolution 1_05, and 10_1 semi-Lagrangian schemes. The last schemes are the non-filtered semi-Lagrangian schemes with resolution 10_1, 05_05, 3_1, and 1_1.

When considering the ranking of the individual errors the result is quite different. For the rank of the $l_{1}$ error the four best performing schemes are the LMCSL, the classical semiLagrangian, the LMC cascade and the cascade, all filtered and with resolution 1_05.

The second error norm, the rank of the $l_{2}$ error, gives the best correlation with the overall rank for the first schemes. The best performing schemes are the high resolution ASD, the coarse resolution ASD, the filtered fine resolution ASD, and the fourth is the filtered classical semi-Lagrangian scheme using resolution 1_05.

With regard to the rank of the $l_{\infty}$ error, the best performing schemes are the coarse resolution ASD, the fine resolution ASD, the filtered coarse resolution ASD, and fourth is the filtered classical semi-Lagrangian scheme using resolution 10_1.

\subsubsection{Total rank of all test cases}

The last table, Table 7, shows the sum of the total rank of all six test cases. It is seen that the filtered ASD scheme with the finest resolution gets the best score. The second best scheme is SL with filter using resolution 10_1, third is ASD with resolution 05_05, followed by the LMCSL scheme using resolution 10_1, and the two filtered semi-Lagrangian schemes with resolution 1_05. This is followed by the remaining ASD and the last two filtered semi-Lagrangian schemes using resolution 1_05. Generally, the filtered semi-Lagrangian schemes are seen to perform better than the non-filtered solutions, and the order of the five resolutions are 1_05 and 10_1 filtered, 1_05 non-filtered, 05_05 and 3_1 filtered, 05_05 and 10_1 non-filtered, 1_1 filtered, and 3_1 and 1_1 non-filtered.

\subsubsection{Comparison of the ranking table results}

In an overall view, the ASD schemes perform very well when considering the tables above. However, from Table 7 it is seen that the filtered semi-Lagrangian schemes with high time step relative to grid size perform best of the semiLagrangian schemes. Some of these schemes are even better than the ASD schemes run with coarse resolution. One expects that the coarse resolution semi-Lagrangian schemes often perform better than the other resolutions because these schemes introduce interpolation errors when calculating concentrations in the departure points. In the coarse resolution results given here, only half as many or fewer interpolations are carried out, and thereby less overall error is introduced.

\subsection{Timing and optimization}

The code of the methods presented in this work has not been optimized. Therefore, the time a model run uses does not tell whether the method is faster than DEHM, which has already been optimized to run on parallel computers.

It should be noted that the modified interpolation weights in practice only should be calculated once every time step rather than for every single tracer. The semi-Lagrangian schemes perform well for larger time steps (i.e. $C>1$ ), when not considering tests including deposition. Also, since the semi-Lagrangian schemes are local, as opposed to the global ASD, the benefit from optimisation of the code for parallel computation with distributed memory is very likely to be significant.

\section{Discussion, conclusion and future aspects}

\subsection{Discussion}

In Sect. 4.2, the ten desirable properties were introduced. Below is a list of the same properties, with boldface indicating which properties this work fulfills:

- Accuracy

- Stability

- Computational efficiency

- Transportivity and locality

- Shape preservation

- Conservation

- Consistency

- Compatibility

- Preservation of constancy

- Preservation of linear correlations between constituents

The properties fulfilled by the schemes considered in this work are accuracy, stability, transportivity and locality, shape preservation, (mass) conservation, and consistency of the discretization. The differences between a traditional semiLagrangian scheme and the other LMC semi-Lagrangian and 
cascade methods proposed here are conservation and consistency of the discretization. The stability can be seen from the plots of the slotted cylinder with various time steps. The method is not dependent on the size of the time steps, but on the number of time steps. The applied filter imposes shape preservation when pure advection is considered, however, it is not computationally efficient. When chemistry and advection are combined the filter can no longer ensure shape preservation due to the non-linearities in the chemistry. The semi-Lagrangian method is optimal when considering advection using large Courant numbers, because less interpolation noise would be introduced. Besides, in air pollution modelling, using a large time step relative to wind speed (meaning greater than the CFL condition) may cause problems related to the emission and deposition processes. In case of emissions, a large semi-Lagrangian time step would cause the advection to jump over some grid cells and, therefore, to miss the contribution from specific emissions to the concentration field unless the emissions are taken into account in another way. Something similar happens when considering deposition.

The semi-Lagrangian schemes using the modified interpolation weights are considered efficient, see Kaas (2008), however, when adding the locally mass conserving and monotonic filter, twice the computational power is needed to perform the calculations. Therefore, the filter is not considered to be computationally efficient, see Kaas and Nielsen (2010). It still remains to be seen whether the LMCSL and LMC cascade methods combined with the filter are more computationally efficient than ASD.

With regard to interpolation method in the semiLagrangian scheme, classical bi-cubic interpolation versus cubic cascade interpolation, it can be seen from the plots and error measure tables that the difference between the results is insignificant if any. The advantage of choosing cascade interpolation over classical semi-Lagrangian is that the interpolation would be slightly faster due to the dependence on fewer departure points. On the other hand, this method demands slightly more memory for calculation of the intermediate time step.

It would be interesting to see how the timings of the semiLagrangian schemes are relative to ASD, which is already optimized for parallel computing. This will be tested in future work.

\subsection{Conclusion}

The aim of the present work has been to test new semiLagrangian models against the method used in the Danish Eulerian Hemispheric Model (DEHM) currently run at the National Environmental Research Institute (NERI) in Denmark. The semi-Lagrangian (SL) methods combine the classical cubic interpolation with cascade interpolation, see Nair et al. (2002), the modified interpolation weights by Kaas (2008) and the locally mass conserving monotonic filter by
Kaas and Nielsen (2010). The methods were tested on conditions representing both rural and urban chemistry. The urban chemistry introduces steeper gradients than the rural chemistry and is therefore a harder test for the advection schemes. Ranking of calculated error measures compares the general performance of the schemes.

It was seen that the Accurate Space Derivative (ASD) method used in DEHM performs very well when considering the cosine hill. For the semi-Lagrangian methods it was seen that the filter improves the results, however, when combining chemistry and advection the resulting shape of the cone, in case of $\mathrm{NO}_{2}$, was a little altered. For $\mathrm{O}_{3}$, the differences between ASD and the SL schemes are significant. The overshoots generated by ASD for urban chemistry are only exceeded by the filtered solutions of the SL methods, whereas only the SL schemes generate undershoots.

Various tests using the slotted cylinder were also performed to test the ability of the schemes to model steep gradients. It was seen that only for the toughest case, urban chemistry, did the SL schemes outperform the ASD. Visualisations of the rotation tests, show that the error introduced when calculating trajectories in the semi-Lagrangian schemes was insignificant compared to the analytical trajectories. It was also tested at which resolution the filtered LMCSL scheme performed best on the slotted cylinder. As expected, the semiLagrangian schemes perform best when using a fine resolution with a large time step. This is due to the errors introduced by the interpolation. The ranking showed that the filtered solutions of the SL schemes perform better than the non-filtered solutions and in some cases even better than the ASD scheme (e.g. resolution 10_1 and 1_05 when considering the slotted cylinder with urban chemistry).

The sum of the rank(all) shows that some of the filtered semi-Lagrangian schemes using resolution 1_05 and 10_1 performed better than ASD with resolution 1_1. The best performing scheme is ASD with resolution 05_05. However, comparing results with different spatial resolution is not straight forward, because a finer spatial resolution in air pollution models will give steeper gradients for the method to model given the sharper gradients in the emissions. It might not be advantageous to increase the grid resolution in DEHM when compared to the expense in computational efficiency. Increasing the number of grid cells to twice as many in both $\mathrm{x}$ - and $\mathrm{y}$-directions would make the model eight times as computational expensive to run.

Overall, the ASD is the best performing scheme in most of the cases. The semi-Lagrangian filtered solutions were good, especially for sharp gradients and the non-filtered solutions were good, or the best performing SL schemes, when considering the rotating cone and $\mathrm{O}_{3}$. The difference between classical semi-Lagrangian and cascade interpolation and using the modified interpolation weights or not is insignificant when considering the ranking tables, however, the properties of mass conservation and computational efficiency are 
important in air pollution models. Active chemistry enhances the errors in the advection due to non-linearities.

Adding the locally mass conserving monotonic filter to the semi-Lagrangian methods improves the results. It would be interesting to see how other filters would influence the results. It is, however, important to keep in mind that the semiLagrangian methods with or without the filter are local, one of the desirable properties for advection schemes. On the other hand, the filter might not computationally efficient, see Kaas and Nielsen (2010).

In conclusion, the semi-Lagrangian schemes using resolution 1_1 and 3_1 are not able to outperform the ASD using resolution 1_1, based on the studies presented in this work, therefore the hypothesis is rejected. However, using other resolutions or e.g. higher order interpolation techniques it might be possible for the semi-Lagrangian schemes to outperform the ASD scheme. Also, there are advantages with the semi-Lagrangian methods, namely, they are local (an advantage in parallellisation), locally mass conserving, stable for longer time steps, and finally the semi-Lagrangian methods are generally better at handling sharp gradients in time and space than traditional Eulerian methods.

\subsection{Future aspects}

A current problem for the stable very long time stepping in semi-Lagrangian methods is treatment of deposition and emissions. A possible solution would be to consider emissions and depositions along the trajectories. A future aim would be to investigate how much more calculation time this would require.

Generally, optimisation of the semi-Lagrangian schemes for parallel computing with distributed memory should be relatively straightforward because the semi-Lagrangian schemes are local as opposed to ASD which is a global method. This should also make the parallelisation more efficient. With regard to filters, the future optimisation should focus on mass conservation, monotonicity and computational efficiency. Furthermore, it is important that the filters are not too efficient.

An algorithm for calculation of emissions and deposition along trajectories has not been developed yet, but the time spent on the development might easily be earned back from gain in computational efficiency from possible increase in size of time step. Since dry deposition is taken as the lower boundary and emissions are only in the lowest levels of the atmosphere, the most time consuming part would be wet deposition which may occur throughout the atmosphere.

It still remains to be seen how the new proposed method performs when implemented in a real air pollution model. It is important to have a high accuracy advection scheme, which at the same time is very efficient computationally.
Acknowledgement. The present study is a part of the research of the "Center for Energy, Environment and Health", financed by The Danish Strategic Research Program on Sustainable Energy under contract no 2104-06-0027.

Edited by: A. Sandu

\section{References}

Bartnicki, J.: A Simple Filtering Procedure for Removing Negative Values from Numerical Solutions of the Advection Equation, Environ. Softw., 4, 187-201, 1989.

Bott, A.: A Positive Definite Advection Scheme Obtained by Nonlinear Renormalization of the Advective Fluxes, Mon. Weather Rev., 117, 1006-1015, 1989.

Brandt, J.: Modelling Transport, Dispersion and Deposition of Passive Tracers from Accidental Releases, PhD thesis, Ministry of Environment and Energy National Environmental Research Institute and Ministry of Research and Information Technology Ris $\emptyset$ National Laboratory, 307 pp., 1998.

Brandt, J., Dimov, I., Goergiev, K., Wasniewski, J., and Zlatev, Z.: Coupling the Advection and the Chemical Parts of Large Air Pollution Models, Lecture Notes in Computer Science, Applied Parallel Computing, Industrial Computation and Optimization, 1184, 65-76, 1996a.

Brandt, J., Wasniewski, J., and Zlatev, Z.: Handling the Chemical Part in Large Air Pollution Models, Appl. Math. Comp. Sci., 6, 331-351, 1996b.

Christensen, J. H.: Transport of Air Pollution in the Troposphere to the Artic, Ph.D. thesis, Ministry of Environment and Energy National Environmental Research Institute and Ministry of Research and Information Technology Ris $\varnothing$ National Laboratory, 377 pp., 1995.

Christensen, J. H.: The Danish Eulerian Hemispheric Model - A Three-Dimensional Air Pollution Model used for the Artic, Atmos. Environ., 31, 4169-4191, 1997.

Crowley, W. P.: Numerical Advection Experiments, Mon. Weather Rev., 96, 1-11, 1968.

Dabdub, D. and Seinfeld, J. H.: Numerical Advective Schemes used in Air Quality Models - Sequential and Parallel Implementation, Atmos. Environ., 28, 3369-3385, 1994.

Durran, D. R.: Numerical Methods for Wave equations in Geophysical Fluid Dynamics, Springer, pp. 465, 1999.

Flatøy, F. and Hov, Ø.: Three-dimensional model studies of the effect of $\mathrm{NO}_{\mathrm{x}}$ emissions from aircraft on ozone in the upper troposphere over Europe and the North Atlantic, J. Geophys. Res., 101, 1401-1422, 1996.

Forester, C. K.: Higher Order Monotonic Convective Difference Schemes, J. Comput. Phys., 23, 1-22, 1977.

Frohn, L. M.: A study of long-term high-resolution air pollution modelling, $\mathrm{PhD}$ thesis, University of Copenhagen and National Environmental Research Institute, 444 pp., 2004.

Frohn, L. M., Christensen, J. H., and Brandt, J.: Development of a High-Resolution Nested Air Pollution Model, J. Comput. Phys., 179, 68-94, 2002.

Frohn, L. M., Christensen, J. H., Brandt, J., Geels, C., and Hansen, K. M.: Validation of a 3-D hemispheric nested air 
pollution model, Atmos. Chem. Phys. Discuss., 3, 3543-3588, doi:10.5194/acpd-3-3543-2003, 2003.

Hov, Ø., Zlatev, Z., Berkowicz, R., Eliassen, A., and Prahm, L. P.: Comparison of Numerical Techniques for use in Air Pollution Models with Non-Linear Chemical Reactions, Atmos. Environ., 23, 967-983, 1989.

Jonson, J., Kylling, A., Berntsen, T., Isaksen, I., Zerefos, C. S., and Kourtidis, K.: Chemical effects of UV fluctuations inferred from total ozone and tropospheric aerosol variations, J. Geophys. Res., 105, 14561-14574, 2000.

Kaas, E.: The Construction of and tests with a multi-level, semiLagrangian and semi-implicit limited area model, A HIRLAM technical report and Master degree thesis, Danish Meteorological Institute, 1987.

Kaas, E.: A simple and efficient locally mass conserving semiLagrangian transport scheme, Tellus, 60, 305-320, 2008.

Kaas, E. and Nielsen, J.: A Mass Conserving Quasi-Monotonic Filter for Use in Semi-Lagrangian Models, Mon. Weather Rev., 138(5), 1858-1876, doi:10.1175/2009MWR3173.1, 2010.

Kylling, A., Bais, A. F., Blumthaler, M., Schreder, J., Zerefos, C. S., and Kosmidis, E.: The effect of aerosols on solar UV irradiances during the Photochemical Activity and Solar Radiation campaign, J. Geophys. Res., 103, 26051-26060, 1998.

Machenhauer, B., Kaas, E., and Lauritzen, P. H.: Handbook on Numerical Analysis - Finite Volume Methods in Meteorologi, Elsevier, 2008.
Molenkamp, C. R.: Accuracy of Finite-Difference Methods Applied to the Advection Equation, J. Appl. Meteorol., 7, 160-167, 1968.

Nair, R. D., Scroggs, J. S., and Semazzi, F. H. M.: Efficient Conservative Global Transport Schemes for Climate and Atmospheric Chemistry Models, Mon. Weather Rev., 130, 2059-2073, 2002.

Rasch, P. J. and Williamson, D. L.: Computational aspects of moisture transport in global models of the atmosphere, Q. J. Roy. Meteorol. Soc., 116, 1071-1090, 1990.

Robert, A.: A Stable Numerical Integration Scheme for the Primitive Meteorological Equations, Atmos. Ocean, 19, 35-46, 1981.

Simpson, D., Fagerli, H., Jonson, J. E., Tsyro, S., Wind, P., and Tuovinen, J.-P.: Transboundary Acidification, Eutrophication and Ground Level Ozone in Europe, Part 1., Tech. rep., Norwegian Meteorological Institute, available at: www.emep.int (last access date: 20 June 2011), 2003.

Sun, W.-Y. and Yeh, K.-S.: A General semi-Lagrangian Advection Scheme Employing Forward Trajectories, Q. J. Roy. Meteorol. Soc., 123, 2463-2476, 1997.

Zerroukat, M., Wood, N., and Staniforth, A.: SLICE: A SemiLagrangian Inherently Conserving and Efficient scheme for transport problems, Q. J. Roy. Meteorol. Soc., 128, 2801-2820, 2002.

Zerroukat, M., Wood, N., and Staniforth, A.: SLICE-S: A SemiLagrangian Inherently Conserving and Efficient scheme for transport problems on the sphere, Q. J. Roy. Meteorol. Soc., 130, 2649-2664, 2004. 